\title{
Higher-order Lagrangian systems: Geometric structures, dynamics, and constraints
}

\author{
X. Gràcia \\ Departament d'Estructura i Constituents de la Matèria, Universitat de Barcelona, Facultat de Fisica, \\ Departament de Matemàtica Aplicada i Telemàtica, Av. Diagonal, 647, E-08028 Barcelona, \\ Spain, Universitat Politècnica de Catalunya, ETSE Telecomunicació, C/Jordi Girona Salgado s/ $n, E-$ \\ 08034 Barcelona, Spain \\ J.M. Pons ${ }^{\text {a) }}$ \\ Departament d'Estructura i Constituents de la Materia, Universitat de Barcelona, Faultiat de Fitsica, \\ Av. Diagonal, 647, E-08028 Barcelona, Spain \\ N. Román-Roy \\ Departament de Matemàtica Aplicada i Telemàtica, Universitat Politècnica de Catalunya. \\ ETSE Telecomunicació, C/ Jordi Girona Salgado s/n, E-08034 Barcelona, Spain \\ (Received 17 January 1990; accepted for publication 30 April 1991)

\begin{abstract}
In order to study the connections between Lagrangian and Hamiltonian formalisms constructed from a-perhaps singular-higher-order Lagrangian, some geometric structures are constructed. Intermediate spaces between those of Lagrangian and Hamiltonian formalisms, partial Ostrogradskiî's transformations and unambiguous evolution operators connecting these spaces are intrinsically defined, and some of their properties studied. Equations of motion, constraints, and arbitrary functions of Lagrangian and Hamiltonian formalisms are thoroughly studied. In particular, all the Lagrangian constraints are obtained from the Hamiltonian ones. Once the gauge transformations are taken into account, the true number of degrees of freedom is obtained, both in the Lagrangian and Hamiltonian formalisms, and also in all the "intermediate formalisms" herein defined.
\end{abstract}

\section{INTRODUCTION}

The Lagrangian and Hamiltonian formulations of dynamical systems are the natural framework for most of the developments in theoretical physics, and have been a subject of increasing research for a long time. Here, we want to point out two directions of development of these formalisms: (a) the extension to the singular case (i.e., when the Hessian matrix of the Lagrangian with respect to the velocities is singular), and (b) the generalization to the higher-order case (i.e., with Lagrangians depending upon the $k$ th derivative of coordinates-or fields if we are dealing with field theory).

Both developments are relevant in theoretical physics. Lagrangians for gauge theories-the basis of BRST symmetry-are necessarily singular, due to the arbitrariness underlying gauge transformations. On the other hand, several physical theories use higher-order Lagrangians. This is the case, for instancc, of Hilbert's action for gravitation [Refs. 1, 2 (references therein), 3, 4], the recent Polyakov's formulation of the string [Refs. 5, 6 (references therein)], or Podolski's electrodynamics and its generalizations to YangMills theories ${ }^{7-10}$ - see also Refs. 11-16 and references in Ref. 17. Finally, the field-antifield formalism of Batalin and Vilkovisky usually leads to higher-order Lagrangians. ${ }^{18}$

About 1950, Dirac and Bergmann opened the way for the study of singular Lagrangians and their associated Hamiltonian formalism-see references in Ref. 19, for instance. A lot of work has been made since. Geometrization of the

\footnotetext{
a) Present address: Center for Relativity, Department of Physics, University of Texas at Austin, Austin, TX 78712.
}

regular first-order case was established through the use of the underlying geometric structures of some vector bundles-see Ref. 20, for instance. A bit later, this geometrization was extended to the singular case, together with many results concerning the equivalence of Lagrangian and Hamiltonian formulations and some new relations between both formalisms-see references in Refs. 21-24.

Ostrogradskili, in the middle of the last century, developed the canonical formulation associated to a regular (i.e., nonsingular) Lagrangian of arbitrary finite order. ${ }^{25,3}$ On this basis several theoretical aspects of this formalism have been studied. This includes field theory and quantization, constraints, and equivalence with Lagrangian formalism. $^{26-37}$

Geometrization of higher-order Lagrangian formalism has also been performed, ${ }^{29,38-41}$ as well as the Hamiltonian formalism in the regular case. ${ }^{29}$ Nevertheless, the program to study and geometrize the higher-order singular formalisms is far from being completed. Contribution in this direction is the scope of the present paper.

Recently, ${ }^{33}$ some progress has been made in the secondorder singular case through the introduction of an intermediate space between the spaces of the Lagrangian and Hamiltonian formalisms. With this space several points of the dynamics of these formalisms have been clarified. One of the purposes of the present paper is to pursue this work and also to generalize the method to the $k$-th order case. The Lagrangian may be regular or singular, and only some weak regularity conditions are needed. We work in a finite-dimensional configuration space, but our results can be applied to field theory in the same lines of Refs. 42 and 19.

First, we shall intrinsically define a family of intermedi- 
ate spaces that connect those of Lagrangian and Hamiltonian formalisms, through a decomposition of the LegendreOstrogradskir's transformation.

Related to these "partial Ostrogradskir's transformations" there is a family of unambiguous evolution operators $K_{0}, \ldots, K_{k-1}$. For first-order Lagrangians they have been used to describe the constraints in Lagrangian and Hamiltonian formalisms, ${ }^{43-45}$ and can be given a neat characterization $^{46}$ that will be adopted in the present paper.

The intermediate spaces are suitable for their own formulation of the dynamics, and it turns out that all these formulations are equivalent (they give the same trajectories in configuration space $Q$ ), thus extending the equivalence between the Hamiltonian and the Lagrangian formalisms to all the "intermediate formalisms."

When dealing with a singular Lagrangian all these dynamics are defined by a differential equation that cannot be written in normal form. This is the well-known situation of constrained systems and leads to stabilization algorithms in order to determine-partially, at least-the dynamics, which had a certain degree of arbitrariness due to gauge freedom, and to find out the submanifold where the motion can take place.

All these constructions can be performed for any $k$ thorder Lagrangian function. Although they may seem rather formal, they are really useful, especially for singular Lagrangians. The aim of the intermediate formalisms is to relate the dynamics of Lagrangian and Hamiltonian formalisms. Their equivalence can be proven directly ${ }^{36}$ but in view of computations it is more interesting to relate their constraints. As it will be shown in the present paper, these constraints can be obtained through the application of the intermediate evolution operators. In particular, the Lagrangian constraints are obtained by consecutively applying the $k$ evolution operators $K_{k-1}, \ldots, K_{0}$ to the Hamiltonian constraints. The proof of this result, as well as the construction of the operators, strongly relies on the introduction of the intermediate spaces and their dynamics. They are also applied to study the arbitrary functions appearing in the dynamics.

Once the constraints and the dynamics have been determined, a gauge fixing procedure can be introduced in order to identify the physical degrees of freedom; their number, which is actually computed, is the same in all the spaces. The Lagrangian gauge fixing is also derived from the Hamiltonian one, again through the use of the intermediate evolution operators.

The paper is organized as follows. In Sec. II some concepts on higher-order tangent bundles are introduced. Section III is devoted to some general remarks on higher-order Lagrangians. The intrinsic definition of the "intermediate spaces" is given in Sec. IV, and the "partial Ostrogradskir's transformations" in Sec. V, together with some of their properties. Sections VI and VII are devoted to the evolution operators and some relations between them. Some special features of the space of the Hamiltonian formalism, including its primary constraints, are studied in Sec. VIII. A closer knowledge on the partial Ostrogradskiî's transformations is thus obtained in Sec. IX.

The "Lagrangian" equations of motion in each space $P_{r}$ for $0 \leqslant r \leqslant k-1$ are introduced for paths in Sec. $\mathrm{X}$ and vector fields in Sec. XI. The Hamilton-Dirac equations of motion for a path in $P_{r}$ are introduced in Sec. XII. Various relations between the dynamical vector fields and the unambiguous evolution operators are obtained in Sec. XIII, and some particular cases of them are pointed out in the following section. Then we are ready to develop the stabilization algorithms in Sec. XV, along with the determination of the "arbitrary" functions in Sec. XVI. Section XVII deals with the gauge fixing procedure and the number of degrees of freedom. We finish with examples and conclusions.

Our notations and conventions are similar to those of Refs. 29, 33, and 47. All manifolds and mappings are assumed to be $C^{\infty}$. Indices of coordinates are generally omitted, and the summation convention is assumed for them.

In order to apply the stabilization algorithm for Hamiltonian formalism, ${ }^{43}$ and the Ostrogradskil's theorem for singular Lagrangians, ${ }^{36}$ the following regularity conditions are assumed: the Hessian matrix $W$ of $L$ has constant rank, ineffective constraints do not appear at any level, and the rank of the matrices of Poisson brackets considered in Sec. XV is constant.

\section{SOME CONCEPTS ON HIGHER-ORDER TANGENT BUNDLES}

In this section we recall some basic concepts and notation concerning higher-order tangent bundles. ${ }^{29,48,49}$ Here and especially in Sec. IV some knowledge of fiber product manifolds will be needed. ${ }^{47,49-51}$

Throughout this paper $Q$ is an $n$-dimensional differentiable manifold. We shall make extensive use of the tangent bundle of order $m \geqslant 0, T^{m}(Q)$, which is an $n(m+1)$-dimensional manifold, whose points are $m$-velocities. These are equivalence classes of curves in $Q$, this equivalcnce being the tangency of order $m$.

If $q^{\circ}$ are coordinates in $Q$, there are natural coordinates $\left(q^{0}, q^{1}, \ldots, q^{m}\right)$ in $T^{m}(Q)$, which represent the first $m$ derivatives of a curve in $Q$. These are the only coordinates that will be considered.

In a natural way, one can define a fiber bundle structure $o_{l}^{m}: T^{m} Q \rightarrow T^{l} Q$ for $m \geqslant l$, that can be written, in natural coordinates,

$$
o_{l}^{m}\left(q^{o}, \ldots, q^{m}\right)=\left(q^{o}, \ldots, q^{l}\right)
$$

From $o_{l}^{m}$ an exact sequence of vector $T^{m} Q$-bundles is constructed

$$
0 \rightarrow V_{l}\left(T^{m} Q\right) \rightarrow T\left(T^{m} Q\right) \stackrel{T\left(o_{i}^{m}\right)}{\rightarrow} T^{m} Q \times_{o_{i}^{m}} T\left(T^{l} Q\right) \rightarrow 0,
$$

which defines the "vertical vectors of order $l$," $V_{l}\left(T^{m} Q\right):=\operatorname{Ker} T\left(o_{l}^{m}\right)$; it is a vector subbundle of $T\left(T^{m} Q\right)$ of rank $n(m-l)$, for which $\partial / \partial q^{l+1}, \ldots, \partial / \partial q^{m}$ constitute a local frame. The coordinate expression of such vectors is $\left(q^{0}, \ldots, q^{m} ; 0, \ldots, 0, v^{l+1}, \ldots, v^{m}\right)$. A section of this subbundle will be called, of course, a vertical field of order $l$.

Moreover, there are the corresponding "horizontal covectors of order $m-l$," the vector subbundle $V_{l}\left(T^{m} Q\right)^{1}$ $\subset T^{*}\left(T^{m} Q\right)$, locally spanned by $d q^{0}, \ldots, d q^{l}$. A one-form $\theta$ in 
$T^{m} Q$ is called horizontal of order $m-l(0 \leqslant l \leqslant m)$ if its range is in this subbundle. The dual sequence of $(2.2)$ is

$0 \rightarrow T^{m} Q \times_{o_{l}^{m}} T\left(T^{l} Q\right)^{*} \stackrel{T\left(o_{l}^{m}\right)}{\rightarrow} T\left(T^{m} Q\right)^{*} \rightarrow V_{l}\left(T^{m} Q\right)^{*} \rightarrow 0$,

from which the isomorphism

$$
T^{m} Q \times_{T^{\prime} Q^{*}} T^{*}\left(T^{l} Q\right) \cong V_{l}\left(T^{m} Q\right)^{\perp} \subset T^{*}\left(T^{m} Q\right)
$$

is deduced. Thus a one-form horizontal of order $m-l$ is identified with a section of the first vector bundle; therefore, with a $T^{l} Q$-bundle morphism $F_{\theta}: T^{m} Q \rightarrow T^{*}\left(T^{l} Q\right)$. If $\theta=A_{0} d q^{0}+\ldots+A_{l} d q^{l}, \quad$ then $\quad F_{\theta}\left(q^{0}, \ldots, q^{m}\right)$ $=\left(q^{0}, \ldots, q^{l} ; A_{0}, \ldots, A_{l}\right)$.

Generalizing the vertical endomorphism of $T(T Q)$, the tangent bundle of $T^{m} Q$ is provided with $m$ "vertical endomorphisms" $J_{r}(1 \leqslant r \leqslant m) . J_{i}$ reads, in coordinates,

$$
J_{1} \circ \frac{\partial}{\partial q^{i-1}}=i \frac{\partial}{\partial q^{i}}
$$

in other words

$$
J_{1}\left(q^{0}, \ldots, q^{m} ; v^{0}, \ldots, v^{m}\right)=\left(q^{0}, \ldots, q^{m} ; 0, v^{0}, 2 v^{1}, \ldots, m v^{m-1}\right) .
$$

The other is obtained from it: $J_{r}=\left(J_{1}\right)^{r}$. Notice that $\operatorname{Ker} J_{r}=\operatorname{Im} J_{m \ldots+1}=V_{m-r}\left(T^{m} Q\right)$.

The transposed endomorphisms ${ }^{t} J_{r}$, will be also considered. They operate in the cotangent bundle of $T^{m} Q$ and their action is given by

$$
{ }^{t} J_{1} \circ d q^{i}=i d q^{i-1}
$$

or, on a point of $T^{*}\left(T^{m} Q\right)$,

$$
{ }^{t} J_{1}\left(q^{0}, \ldots, q^{m} ; p_{0}, \ldots, p_{m}\right)=\left(q^{0}, \ldots, q^{m} ; p_{1}, 2 p_{2}, \ldots, m p_{m}, 0\right) .
$$

Actually we shall need the more general expression

$$
\begin{aligned}
& J_{r}\left(q^{0}, \ldots, q^{m} ; p_{0}, \ldots, p_{m}\right) \\
& \quad=\left[q^{0}, \ldots, q^{m} ;(r ! / 0 !) p_{r}, \ldots,[m ! /(m-r) !] p_{m}, 0, \ldots, 0\right] .
\end{aligned}
$$

Now we have $\operatorname{Ker}^{t} J_{r}=\operatorname{Im}^{t} J_{m-r+1}=V_{r-1}\left(T^{m} Q\right)^{1}$.

There is also a natural closed embedding $j^{m}: T^{m+1} Q \rightarrow T\left(T^{m} Q\right)$, whose local expression is

$$
j^{m}\left(q^{0}, \ldots, q^{m+1}\right)=\left(q^{0}, \ldots, q^{m} ; q^{1}, \ldots, q^{m+1}\right) .
$$

This is, in fact, a vector field along $o_{m}^{m+1}$, and therefore it acts as a differential operator: if $f \in C^{\infty}\left(T^{m} Q\right)$, then

$$
d_{T} f:=\mathscr{L}_{j^{\prime \prime}} f=\left\langle d f, j^{m}\right\rangle
$$

is a function in $T^{m+1} Q$, whose local expression is

$$
d_{T} F=\sum_{i=0}^{m} \frac{\partial f}{\partial q^{i}} q^{i+1}
$$

This notation will be freely used throughout this paper to derivate functions depending on an undetermined number of higher-order velocities.

Finally another particular class of vectors will be considered in $T\left(T^{m} Q\right)$. We say that $v_{g} \in T_{q}\left(T^{m} Q\right)$ satisfies the sth-order condition (for $2 \leqslant s \leqslant m+1$ ) if

$$
T\left(o_{s-2}^{m}\right) \cdot v_{q}=j^{s-2}\left(o_{s-1}^{m}(q)\right) \text {. }
$$

It amounts to say that the coordinate expression of $v_{q}$ has the form $\left(q^{0}, \ldots, q^{m} ; q^{1}, \ldots, q^{s-1}, v^{s-1}, \ldots, v^{m}\right)$. Notice that such vectors constitute an affine subbundle of the vector bundle $T\left(T^{m} Q\right)$, associated to the vector subbundle $V_{s-2}\left(T^{m} Q\right)$. The sth-order condition will be also considered on vector fields of $T^{m} Q$ in an obvious way. Then, a vector field satisfying the $(m+1)$ th-order condition gives rise to a $(m+1)$ th-order differential equation in $Q$.

\section{HIGHER-ORDER LAGRANGIANS}

Now let us consider a $k$ th-order Lagrangian in $Q$, that is to say, a function $L: T^{k} Q \rightarrow \mathbf{R}$. Variational calculus yields its Euler-Lagrange equations $[L]_{\eta(x)}=0$, where

$$
[L]=\sum_{i=0}^{k}(-1)^{i} d_{T}^{i}\left(\frac{\partial L}{\partial q^{i}}\right)
$$

contains derivatives of $q$ up to order $2 k$.

If the solutions of these equations are to be considered as integral curves of a vector field, this should be defined in the manifold $T^{2 k-1} Q$. Although the $k$ th-order Lagrangian formalism can be geometrized in other ways, this is perhaps the better one to build up a Hamiltonian formalism from it. The first step is to construct the Jacobi-Ostrogradskil one-form $\theta_{L}$ in $T^{2 k-1} Q$. It can be defined ${ }^{29}$ by extending the action of $d_{T}$ to one-forms, and its coordinate expression is

$$
\theta_{L}=\sum_{i=0}^{k-1} \hat{p}_{i} d q^{i}
$$

where the $k n$ functions $\hat{p}_{i}$ are the Jacobi-Ostrogradskil momenta

$$
\hat{p}_{i}=\sum_{j=0}^{k-i-1}(-1)^{j} d_{T}^{j}\left(\frac{\partial L}{\partial q^{i+j+1}}\right) .
$$

Notice that $\hat{p}_{i}$ depends at most in $q^{2 k-1-i}$, and we have

$$
\frac{\partial \hat{p}_{i}}{\partial q^{2 k-1 \cdots i}}=(-1)^{k-1-i} W\left(q^{0}, \ldots, q^{k}\right),
$$

where $W$ is the "Hessian matrix"

$$
W:=\frac{\partial^{2} L}{\partial q^{k} \partial q^{k}} .
$$

A very important relation between the momenta is the following:

$$
\hat{p}_{i-1}=\frac{\partial L}{\partial q^{i}}-d_{T} \hat{p}_{i} .
$$

Then, with $\theta_{L}$ one defines the Lagrange two-form $\omega_{L}$ in $T^{2 k-1} Q$ :

$$
\omega_{L}:=-d \theta_{L} .
$$

On the other hand there is the energy function $E_{L}$ in $T^{2 k-1} Q$. It can be defined as $E_{L}=\left\langle\theta_{L}, X\right\rangle-0_{k}^{2 k-1 *}(L)$ for any vector field $X$ in $T^{2 k-1} Q$ satisfying the $(k+1)$ thrrder condition-see also (6.7). Its local expression is

$$
E_{L}=\sum_{i=1}^{k} \hat{p}_{i-1} q^{i}-L\left(q^{0}, \ldots, q^{k}\right) .
$$

And just as in the first-order case, one is lead to consider the vector fields $X$ in $T^{2 k-1} Q$ which satisfy the $2 k$ th-order 
condition

$$
T\left(a_{2 k}^{2 k-\frac{1}{2}}\right) \circ X=j^{2 k-2}
$$

and the "presymplectic" equation

$$
i_{X} \omega_{L}=d E_{L} \text {. }
$$

Both expressions constitute the classical Euler-Lagrange equations for $L$. It should be noticed that, for a regular Lagrangian, (3.9) has one and only one solution, and that it satisfies the $2 k$ th-order condition. For a singular Lagrangian, (3.9) is to be studied in the theory of presymplectic manifolds. 22,52

Since the one-form $\theta_{L}$ is horizontal of order $k$, it induces a morphism of $T^{k-1} Q$-bundles, $F L:=F_{\theta_{L}}: T^{2 k-1} Q \rightarrow T^{*}\left(T^{k-1} Q\right)$, which can be called the Legendre-Ostrogradskiî's transformation of $L$. It is the natural generalization of the Legendre's transformation for a higher-order Lagrangian. Its local expression is, of course,

$$
F L\left(q^{0}, \ldots, q^{2 k-1}\right)=\left(q^{0}, \ldots, q^{k-1} ; \hat{p}_{0}, \ldots, \hat{p}_{k-1}\right),
$$

and, if $\theta$ and $\omega$ are the canonical one- and two-forms of $T^{*}\left(T^{k-1} Q\right)$, then $F L^{*}(\theta)=\theta_{L}$ and $F L^{*}(\omega)=\omega_{L}$.

If $F L$ has constant rank and connected fibers, and its range is a closed submanifold, then the energy function is projectable through it, and a Hamiltonian formalism can be carried on in $T^{*}\left(T^{k-1} Q\right)$. This is (locally) equivalent to the Lagrangian formalism, provided that $L$ satisfies some regularity conditions. ${ }^{36}$

\section{DEFINITION OF THE INTERMEDIATE SPACES}

In a recent paper ${ }^{33}$ a decomposition of the LegendreOstrogradskir's transformation for second-order Lagrangians was naïvely introduced in coordinate language in the following way:

$$
T^{3} Q \stackrel{\alpha_{0}}{\rightarrow} P_{1} \stackrel{\alpha_{1}}{\rightarrow} T^{*}(T Q),
$$

where $\alpha_{0}\left(q^{0}, \ldots q^{3}\right)=\left(q^{0}, q^{1}, q^{2}, \hat{p}_{0}\right)$ and $\alpha_{1}\left(q^{0}, q^{1}, q^{2}, p_{0}\right)$ $=\left(q^{0}, q^{1}, p_{0}, \hat{p}_{1}\right)$ (recall that the definition of $\hat{p}_{1}$ only involves $\left.q^{0}, q^{1}, q^{2}\right)$.

In other words, the Jacobi-Ostrogradskil momenta are introduced step-by-step, and the higher velocities are correspondingly swept off. This decomposition proved to be useful in the analysis of constraints appearing in Lagrangian and Hamiltonian formalisms, and in the determination of the dynamics.

As it was said in the same paper, this has a straightforward generalization to $k$ th-order Lagrangians, $P_{0} \rightarrow{ }^{\alpha_{0}} P_{1} \rightarrow \cdots \rightarrow{ }^{\alpha_{k-1}} P_{k}$, but in no case the structure of such "intermediate spaces" was elucidated.

In order to define the intermediate spaces, we first recall that if $f: M \rightarrow N$ is any manifold morphism, then $M$ is diffeomorphic to its graph $\{(x, y) \in M \times N \mid y-f(x)\}$. Therefore, one is lead to consider, for $0 \leqslant r \leqslant k$, the fiber product manifold

$$
\bar{P}_{r}:=T^{2 k-1-r} Q \times_{T^{k-1} Q^{*}} T^{*}\left(T^{k-1} Q\right)
$$

with natural coordinates $\left(q^{0}, \ldots, q^{2 k-1-r} ; p_{0}, \ldots, p_{k-1}\right)$, hence of dimension $(3 k-r) n$; and locally define $P_{r}$, the "interme- diate space with $r$ momenta," as the subset of $\bar{P}_{r}$ defined by

$$
p_{r}-\hat{p}_{r}=\cdots=p_{k-1}-\hat{p}_{k-1}=0,
$$

which is a $2 k n$-dimensional submanifold of it because the $(k-r) n$ differentials of these functions are linearly independent everywhere. Notice that a set of fairly natural coordinates for $P_{r}$ is provided by

$$
\left(q^{0}, \ldots, q^{2 k-1-r_{;}} p_{0}, \ldots, p_{r-1}\right) ;
$$

these are the only ones that will be considered in $P_{r}$.

The above construction is independent of coordinates. The best way to show it is to make this construction in an intrinsic way.

Let $\theta$ be the canonical one-form of $T^{*}\left(T^{k-1} Q\right)$; its local expression is

$$
\theta=\sum_{i=0}^{k-1} p_{i} d q^{l}
$$

Consider the endomorphism ${ }^{t} J_{r}$ of this manifold, and pull $\theta$ back through it. The result is another one-form $\left({ }^{t} J_{r}\right)^{*}(\theta)$ in $T^{*}\left(T^{k-1} Q\right)$, whose local expression can be easily computed using (2.8):

$$
\left({ }^{t} J_{r}\right)^{*}(\theta)=\sum_{i=0}^{k-1-r} \frac{(i+r) !}{i !} p_{i+r} d q^{i} .
$$

Its pull-back to $\bar{P}_{r}$ through the second projection of this fiber product is a one-form $\theta_{r}$ in $\bar{P}_{r}$, with the same local expression:

$$
\theta_{r}=\operatorname{pr}_{2}^{*}\left(\left({ }^{t} J_{r}\right) *(\theta)\right)=\sum_{i=0}^{k-1-r} \frac{(i+r) !}{i !} p_{i+r} d q^{i}
$$

On the other hand consider the Jacobi-Ostrogradskir one-form $\theta_{L}$ in $T^{2 k-1} Q$. The $r$ th vertical endomorphism of $T\left(T^{2 k-1} Q\right)$-let us denote it also by $J_{r}$-operates on this one-form to yield another one, ${ }^{t} J_{r} \circ \theta_{L}$, with local expression

$$
{ }^{t} J_{r} \circ \theta_{L}=\sum_{i=0}^{k-1-r} \frac{(i+r) !}{i !} \hat{p}_{i+r} d q^{i} .
$$

Now notice that the highest velocity appearing in this expression is $q^{2 k-1-r}$, corresponding to $\hat{p}_{r}$. Since the canonical projection $T^{2 k-1} Q \rightarrow T^{2 k-1-r} Q$ has connected fibers, the above one-form is projectable through it, and has the same local expression in the last manifold. The result is finally pulled back to $\bar{P}_{r}$ through its first projection, thus obtaining a one-form $\hat{\theta}_{r}$ in $\bar{P}_{r}$, with the same local expression:

$$
\begin{aligned}
\hat{\theta}_{r} & =\operatorname{pr}_{1}^{*}\left(\left(o_{2 k-1-r}^{2 k-1}\right)_{*}\left({ }^{t} J_{r} \circ \theta_{L}\right)\right) \\
& =\sum_{i=0}^{k-1-r} \frac{(i+r) !}{i !} \hat{p}_{i+r} d q^{i} .
\end{aligned}
$$

The local expressions of $\theta_{r}$ and $\hat{\theta}_{r}$ are similar, but $p_{i}$ have been changed to $\hat{p}_{i}(r \leqslant i \leqslant k-1)$. Therefore, we have the following.

Theorem 1: For $0 \leqslant r \leqslant k$ let $P_{r}$ be the subset of $\bar{P}_{r}$ where $\theta_{r}$ and $\hat{\theta}_{r}$ coincide. This is a closed submanifold of dimension $2 k n$, locally defined by the vanishing of the $n(k-r)$ functions $p_{r}-\hat{p}_{r}, \ldots, p_{k-1}-\hat{p}_{k-1}$.

We shall refer to $P_{r}$ as the rth intermediate space. Notice that $P_{0}$ is nothing but the graph of the Legendre-Ostrogradskiı̌'s transformation, therefore it is identified with 
$T^{2 k-1} Q$. On the other hand $P_{k}=\vec{P}_{k}=T^{k-1} Q \times{ }_{T^{k-1} Q} T^{*}\left(T^{k-1} Q\right)$, which is canonically diffeomorphic to $T^{*}\left(T^{k-1} Q\right)$. We shall make both identifications when convenient.

Since $\bar{P}_{r}$ is fibered over $T^{2 k-1-r} Q$, the restriction of its projection to $P_{r}$ defines a mapping

$$
\gamma_{r}: P_{r} \rightarrow T^{2 k-1-r} Q
$$

with local expression

$$
\gamma_{r}\left(q^{0}, \ldots, q^{2 k-1-r^{2}} ; p_{0}, \ldots, p_{r-1}\right)=\left(q^{0}, \ldots, q^{2 k-1-r}\right) ;
$$

this defines a fiber bundle structure in $P_{r}$ over $T^{2 k-1-r} Q$.

We finish by pointing out that our method has been extended to higher-order field theories by Saunders and Crampin, ${ }^{53}$ and that a different construction of intermcdiate spaces has been developped by Cariñena and López. ${ }^{54}$

\section{THE PARTIAL OSTROGRADSKII'S TRANSFORMATIONS}

The previous construction is only justified because the "partial Ostrogradskiil's transformations"-as looked forfit naturally with it, as we are going to prove.

First notice that the mapping $o_{2 k-2}^{2 k-r}: T^{2 k-1-r} Q \rightarrow T^{2 k-2-r} Q$ induces in a natural way a canonical projection $\bar{P}_{r} \rightarrow \bar{P}_{r+1}$.

Theorem 2: The canonical projection $\bar{P}_{r} \rightarrow \bar{P}_{r+1}$ induces a mapping $\alpha_{r}: P_{r} \rightarrow P_{r+1}$, for $0 \leqslant r \leqslant k-1$. Its local expression is

$$
\begin{aligned}
& \alpha_{r}\left(q^{0}, \ldots, q^{2 k-1-r} ; p_{0}, \ldots, p_{r-1}\right) \\
& \quad=\left(q^{0}, \ldots, q^{2 k-2-r_{;}} ; p_{0}, \ldots, p_{r-1}, \hat{p}_{r}\right) .
\end{aligned}
$$

$$
\begin{aligned}
& \text { The } \\
& \text { mapping } \\
& \bar{P}_{r} \rightarrow \bar{P}_{r+1} \\
& \left(q^{u}, \ldots, q^{2 k-1-r_{;}} ; p_{0}, \ldots, p_{k-1}\right) \in P_{r} \quad \text { to the }
\end{aligned}
$$

$\left(q^{0}, \ldots, q^{2 k-2-r_{;}} p_{0}, \ldots, p_{k-1}\right)$ and its last $n(k-(r+1))$ momenta satisfy $p_{i}=\hat{p}_{i}$, thus this image is in $P_{r+1}$.

The mapping $\alpha_{r}$ will be called the rth partial Ostrograds$k i$ 's 's transformation. Notice that the Legendre-Ostrogradskiî's transformation $F L$ is identified with the composition $\alpha_{k-1} 0 \ldots \circ \alpha_{0}: P_{0} \rightarrow P_{k}$. We shall use the notation $\alpha_{r s}:=\alpha_{s-1} 0 \ldots \circ \alpha_{r}$, which defines a mapping $P_{r} \rightarrow P_{s}$.

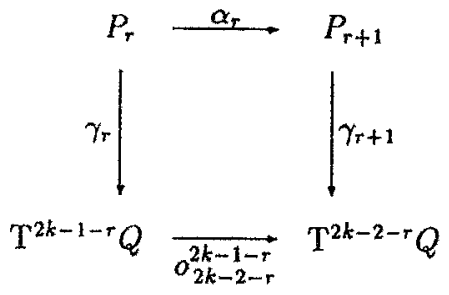

It is clear that the diagram below is commutative:

Now, let us make a closer study of the partial Ostrogradskir's transformation $\alpha_{r}$. The first step is, of course, to compute its tangent mapping. Its action on a coordinate frame is, rather obviously,

$$
\begin{aligned}
& T\left(\alpha_{r}\right) \circ \frac{\partial}{\partial q^{i}}=\frac{\partial}{\partial q^{i}}+\frac{\partial \hat{p}_{r}}{\partial q^{i}} \frac{\partial}{\partial p_{r}}, \quad 0 \leqslant i \leqslant 2 k-2-r \\
& T\left(\alpha_{r}\right) \circ \frac{\partial}{\partial q^{2 k-1-r}}=\frac{\partial \hat{p}_{r}}{\partial q^{2 k-1-r}} \frac{\partial}{\partial p_{r}} \\
& T\left(\alpha_{r}\right) \circ \frac{\partial}{\partial p_{i}}=\frac{\partial}{\partial p_{i}}, \quad 0 \leqslant i \leqslant r-1
\end{aligned}
$$

The Jacobian matrix can be written as a block matrix, each block being a square matrix of order $n$ :

$$
\left(\begin{array}{cccccccc}
I_{n} & & & & 0_{n} & 0_{n} & \cdots & 0_{n} \\
& \ddots & & & \vdots & \vdots & & \vdots \\
& & \ddots & & \vdots & \vdots & & \vdots \\
& & & I_{n} & 0_{n} & 0_{n} & \cdots & 0_{n} \\
0_{n} & \cdots & \cdots & 0_{n} & 0_{n} & I_{n} & & \\
\vdots & & & \vdots & \vdots & & \ddots & \\
0_{n} & \cdots & \cdots & 0_{n} & 0_{n} & & & I_{n} \\
\partial \hat{p}_{r} / \partial q^{0} & \cdots & \cdots & \cdots & \partial \hat{p}_{r} / \partial q^{2 k-1-r} & 0_{n} & \cdots & 0_{n}
\end{array}\right) .
$$

Recalling (3.3) it is clear that the corank of this matrix is the same as the Hessian matrix $W$, and their determinants are related by $\operatorname{det} T\left(\alpha_{r}\right)=(-1)^{r+n(k-1-r)} \operatorname{det} W$. Therefore, the following is shown.

Proposition 1: If any of the partial Ostrogradskili's transformations has constant rank, all them have, it is the same and can be evaluated as

$$
\text { rank } \alpha_{r}=(2 k-1) n+\operatorname{rank} W,
$$

where $W$ is the Hessian matrix of $L$ in any coordinates.
We recall that $\alpha_{r}$ has locally constant rank if and only if $n$ - rank $W$. Notice also that the rank of $F L$ at each point is at least $k(n+\operatorname{rank} W)$. However, $F L$ may not have constant rank even though the $\alpha_{r}$ do.

The canonical symplectic form $\omega$ of $P_{k}=T^{*}\left(T^{k-1} Q\right)$ allows the construction of

$$
\omega_{r}:=\alpha_{r k}^{*}(\omega),
$$

which is an exact two-form in $P_{r}(0 \leqslant r \leqslant k)$, with local Ker $T\left(\alpha_{r}\right)$ is a vector subbundle of $T\left(P_{r}\right)$; then its rank is 
expression

$$
\begin{aligned}
\omega_{r}= & d q^{0} \wedge d p_{0}+\cdots+d q^{r-1} \wedge d p_{r-1} \\
& +d q^{r} \wedge d \hat{p}_{r}+\cdots+d q^{k-1} \wedge d \hat{p}_{k-1} .
\end{aligned}
$$

(In spite of the notation chosen, this $\omega_{r}$ has little to do with the previously constructed one-form $\theta_{r}$.) Notice that $\omega_{0}$ and $\omega_{k}$ are $\omega_{L}$ and $\omega$, respectively, and that

$$
\alpha_{r}^{*}\left(\omega_{r+1}\right)=\omega_{r} .
$$

By inner contraction with tangent vectors at $x \in P_{r}$ a vector $P_{r}$-bundle morphism $\Omega_{r}: T\left(P_{r}\right) \rightarrow T\left(P_{r}\right) *$ is obtained:

$$
\Omega_{r} \cdot v_{x}:=i_{v_{x}} \omega_{r} .
$$

If it has locally constant rank then $\omega_{r}$ is a presymplectic form. If it is an isomorphism then $\omega_{r}$ is a symplectic form.

Now, as in the first-order Lagrangian case, we are ready to characterize the "regularity" or the "singularity" of the Lagrangian in the following sense.

Theorem 3: The following properties are equivalent $(0 \leqslant r \leqslant k-1)$ : (1) The Legendre-Ostrogradskiř's transformation is a local diffeomorphism; (2) $\alpha_{r}$ is a local diffeomorphism; (3) $\omega_{r}$ is a symplectic form; (4) the Hessian matrix $W$ of $L$ is invertible in any coordinates.

Equivalence between (2) and (4) follows from the preceding proposition. The Jacobian of $F L$ is $\pm(\operatorname{det} W)^{k}$, thus (1) and (4) are equivalent. Since $\omega$ is symplectic, (2) implies (3). Finally one should compute

$$
\begin{aligned}
\left(\omega_{r}\right)^{\wedge n k}= & (n k) !(-1)^{k-r+[(k-r) / 2] n} \\
& \times(\operatorname{det} W)^{k-r} d q^{0,1} \wedge \cdots \wedge d p_{r-1, n},
\end{aligned}
$$

to conclude (4) from ( 3 ). (In this expression $q$ 's and $p$ 's bear a second index corresponding to coordinates in $Q$.) The reader should bear in mind that the Hessian matrix depends only in $q^{0}, \ldots, q^{k}$, and that the domains of the partial Ostrogradskir’'s transformations all are $T^{k} Q$-bundles.

If the Lagrangian $L$ satisfies the properties of the theorem then it is called regular. Otherwise it is called singular.

\section{THE INTERMEDIATE EVOLUTION OPERATORS}

As well as in the first-order Lagrangian case ${ }^{43.44}$ it is useful to define an evolution operator $K_{r}$ such that applied to a function in $P_{r+1}$ its time derivative is obtained in $P_{r}$.

By generalizing the second-order case, ${ }^{33}$ we write

$$
\begin{aligned}
K_{r}= & q^{1} \frac{\partial}{\partial q^{0}}+\cdots+q^{2 k-1-r} \frac{\partial}{\partial q^{2 k-2-r}}+\left(\frac{\partial L}{\partial q^{0}}\right) \frac{\partial}{\partial p_{0}} \\
& +\left(\frac{\partial L}{\partial q^{1}}-p_{0}\right) \frac{\partial}{\partial p_{1}}+\cdots+\left(\frac{\partial L}{\partial q^{r}}-p_{r-1}\right) \frac{\partial}{\partial p_{r}} .
\end{aligned}
$$

The analysis of Ref. 46 shows that these operators should be considered as vector fields along the partial Ostrogradskiř's transformations $\alpha_{r}$-see also Ref. 49 for more information on vector fields along mappings. In that paper it is proven that $K$ (in the first-order case) is intrinsically characterized as the vector field along $F L$ that obeys a "secondorder condition" and a "presymplectic equation." To be precise, $K$ is the only mapping $K: T Q \rightarrow T\left(T^{*} Q\right)$ such that

$$
\begin{aligned}
& o_{T^{*} Q} \circ K=F L, \\
& T\left(0_{Q}^{*}\right) \circ K=I d_{T Q}, \\
& F L^{*}\left(i_{K} \omega\right)=d E_{L} .
\end{aligned}
$$

Now let us do the same with $K_{r}$. It should be a vector field along $\alpha_{r}$, that is to say, a lift of $\alpha_{r}$ to $T\left(P_{r+1}\right)$. The diagram below should be commutative:

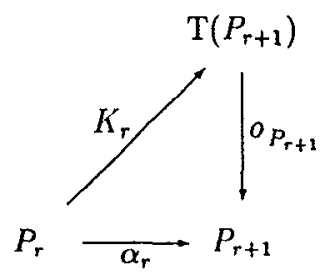

Then, as explained in Ref. $46, K_{r}$ acts as a differential operator on functions $f \in C^{\infty}\left(P_{r+1}\right)$ :

$$
K_{r} \cdot f:=\left\langle d f \circ \alpha_{r}, K_{r}\right\rangle .
$$

In our coordinates for the intermediate spaces, the local expression for such a mapping is

$$
\begin{aligned}
& K_{r}\left(q^{0}, \ldots, q^{2 k-1-r} ; p_{0}, \ldots, p_{r-1}\right) \\
&=v^{0} \frac{\partial}{\partial q^{0}}+\cdots+v^{2 k-2-r} \frac{\partial}{\partial q^{2 k-2-r}} \\
&+w_{0} \frac{\partial}{\partial p_{0}}+\cdots+w_{r} \frac{\partial}{\partial p_{r}},
\end{aligned}
$$

where $v^{0}, \ldots, w_{r}$ are functions in $P_{r}$.

Now consider the following diagram:

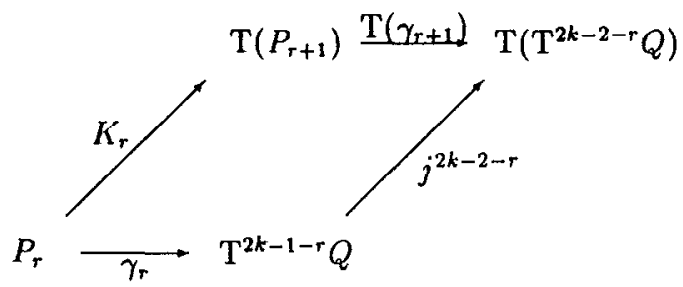

Requiring its commutativity amounts to equating the expressions

$$
\begin{aligned}
& T\left(\gamma_{r+1}\right) \circ K_{r}=\left(q^{0}, \ldots, q^{2 k-2-r^{2}} ; v^{0}, \ldots, v^{2 k-2-r}\right), \\
& j^{2 k-2-r_{\circ}} \gamma_{r}=\left(q^{0}, \ldots, q^{2 k-2-r_{r}} ; q^{1}, \ldots, q^{2 k-1-r}\right) \text {, }
\end{aligned}
$$

that is to say, to determining the functions $v^{0}=q^{1}, \ldots, v^{2 k-2-r}=q^{2 k-1-r}$; therefore it can be said that the vectors which are image of $K_{r}$ satisfy the " $(2 k-r)$ thorder condition."

Next take the two-form $\omega_{r+1} \in \Omega^{2}\left(P_{r+1}\right)$ and contract it with $K_{r}$ to obtain a one-form along $\alpha_{r}$, which we write $i_{K_{r}} \omega_{r+1}$, with local expression

$$
\begin{aligned}
i_{K_{r}} \omega_{r+1}= & q^{\mathrm{l}} d p_{0}+\cdots+q^{r+1} d p_{r}+q^{r+2} d \hat{p}_{r+1} \\
& +\cdots+q^{k} d \hat{p}_{k-1}-w_{0} d q^{0}-\cdots-w_{r} d q^{r} \\
& -\left(K_{r} \cdot \hat{p}_{r+1}\right) d q^{r+1}-\cdots-\left(K_{r} \cdot \hat{p}_{k-1}\right) d q^{k-1} .
\end{aligned}
$$

Since $\hat{p}$ 's depend only on $q^{0}$ to $q^{2 k-2-r}$, the action of $K_{r}$ on them is the same as $d_{T}$. Hence, we have the following one- 
form in $P_{r}$ :

$$
\begin{aligned}
\alpha_{r}^{*}\left(i_{K_{r}} \omega_{r+1}\right) & \\
= & q^{1} d p_{0}+\cdots+q^{r+1} d \hat{p}_{r}+\cdots+q^{k} d \hat{p}_{k-1} \\
& -w_{0} d q^{0}-\cdots-w_{r} d q^{r} \\
& -\left(d_{T} \hat{p}_{r+1}\right) d q^{r+1}-\cdots-\left(d_{r} \hat{p}_{k-1}\right) d q^{k-1} .
\end{aligned}
$$

On the other hand, for $0 \leqslant r \leqslant k-1$ we have the energy function $E_{r}$ in $P_{r}$, defined as

$$
E_{r}:=c^{\circ}\left(j^{k-1} \circ o_{k-1}^{2 k-1-r_{0}} \gamma_{r}, \alpha_{r k}\right)-\left(o_{k}^{2 k-1-r_{\circ}} \gamma_{r}\right) *(L) \text {, }
$$

where $c: T\left(T^{k-1} Q\right) \times_{T^{k-1} Q} T^{*}\left(T^{k-1} Q\right) \rightarrow \mathbf{R}$ is the canonical bilinear mapping. Its local expression is, as desired,

$$
\begin{aligned}
E_{r}\left(q^{0}, \ldots, p_{r-1}\right)= & p_{0} q^{1}+\cdots+p_{r-1} q^{r}+\hat{p}_{r} q^{r+1} \\
& +\cdots+\hat{p}_{k-1} q^{k}-L\left(q^{0}, \ldots, q^{k}\right),
\end{aligned}
$$

therefore $\alpha_{r}^{*}\left(E_{r+1}\right)=E_{r}$ and $E_{0}$ is identified with $E_{L}$. Its differential is the one-form in $P_{r}$ :

$$
\begin{aligned}
d E_{r}= & q^{1} d p_{0}+\cdots+q^{r+1} d \hat{p}_{r}+\cdots+q^{k} d \hat{p}_{k-1} \\
& -\left(\frac{\partial L}{\partial q^{0}}\right) d q^{0}-\left(\frac{\partial L}{\partial q^{1}}-p_{0}\right) d q^{1}-\cdots \\
& -\left(\frac{\partial L}{\partial q^{r}}-p_{r-1}\right) d q^{r}-\left(\frac{\partial L}{\partial q^{r+1}}-\hat{p}_{r}\right) d q^{r+1} \\
& -\cdots-\left(\frac{\partial L}{\partial q^{k}}-\hat{p}_{k-1}\right) d q^{k} .
\end{aligned}
$$

Taking the identities ( 3.5 ) into account, it is now clear that equating the just constructed one-forms in $P_{r}$ is equivalent to determining $w_{0}=\partial L / \partial q^{0}, w_{1}=\partial L / \partial q^{1}-p_{0}, \ldots$, $w_{r}=\partial L / \partial q^{r}-p_{r-1}$. Therefore, we have the following. along $\alpha_{r}$,

Theorem 4: There is one and only one vector field $K_{r}$

$$
o_{P_{r+1}} \circ K_{r}=\alpha_{r} \text {, }
$$

such that it satisfies the following two relations: the " $(2 k-r)$ th-order condition"

$$
T\left(\gamma_{r+1}\right) \circ K_{r}=j^{2 k-2-r^{\circ} \circ \gamma_{r}},
$$

and the "presymplectic equation"

$$
\alpha_{r}^{*}\left(i_{K_{r}} \omega_{r+1}\right)=d E_{r} .
$$

Its local expression is given by (6.1).

$K_{r}$ will be called the rth intermediate evolution operator.

Like the classical Euler-Lagrange equations, if $L$ is regular then the $(2 k-r)$ th-order condition can be deduced from the presymplectic equation.

Proposition 2: If $L$ is regular then $K_{r}$ is the only vector field along $\alpha_{r}$ such that $\alpha_{r}^{*}\left(i_{K_{r}} \omega_{r+1}\right)=d E_{r}$, and is given by

$$
K_{r}=T\left(\alpha_{r}\right) \circ \Omega_{r}{ }^{1} \circ d E_{r} .
$$

\section{SOME COMMUTATION RELATIONS BETWEEN THE EVOLUTION OPERATORS}

An alternative construction of the $K_{r}$, which will provide us with a relation between the contiguous $K$ 's, is the following.
First define the last of them, $K_{k-1}$, as before-it is a bit easier. Then the other can be obtained recursively as follows.

Proposition 3: For $1 \leqslant r \leqslant k-1, K_{r-1}$ is the only vector field along $\alpha_{r-1}$ that satisfies the $(2 k-r+1)$ th-order condition and such that

$$
T\left(\alpha_{r}\right) \circ K_{r-1}=K_{r}{ }^{\circ} \alpha_{r-1} \text {. }
$$

That is to say, the diagram below is commutative:

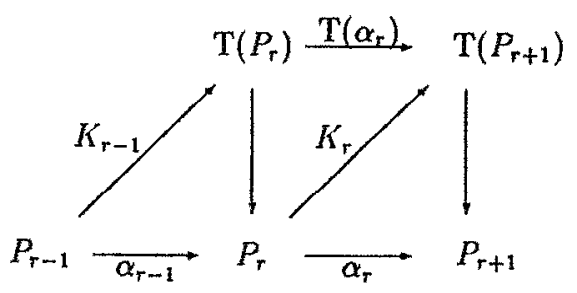

Let us write

$$
K_{r}=\sum_{i=0}^{2 k-2-r} v^{i} \frac{\partial}{\partial q^{i}}+\sum_{j=0}^{r} w_{j} \frac{\partial}{\partial p_{j}}
$$

where the $v^{i}$ and $w_{j}$ are already known.

Also, let

$$
K_{r-1}=\sum_{i=0}^{2 k-1-r} \bar{u}^{-i} \frac{\partial}{\partial q^{i}}+\sum_{j=0}^{r-1} \bar{w}_{j} \frac{\partial}{\partial p_{j}},
$$

where $\bar{v}^{i}$ and $\bar{w}_{j}$ are functions in $P_{r-1}$ to be determined.

Taking (5.2) into account, we obtain

$$
\begin{aligned}
T\left(\alpha_{r}\right) \circ K_{r-1}\left(q^{0}, \ldots, q^{2 k-r_{;} p_{0}, \ldots, p_{r}, 2}\right) \\
=\sum_{i=0}^{2 k-2} \bar{v}^{i} \frac{\partial}{\partial q^{i}}+\sum_{j=0}^{r-1} \bar{w}_{j} \frac{\partial}{\partial p_{j}} \\
\quad+\left(\sum_{i=0}^{2 k-1} \bar{v}^{i} \alpha_{r-1}^{*}\left(\frac{\partial \hat{p}_{r}}{\partial q^{i}}\right)\right) \frac{\partial}{\partial p_{r}} .
\end{aligned}
$$

On the other hand,

$$
\begin{aligned}
K_{r} \circ \alpha_{r-1}\left(q^{0}, \ldots, q^{2 k-r}, p_{0}, \ldots, p_{r-2}\right) \\
=\sum_{i=0}^{2 k-2} \alpha_{r-1}^{*}\left(v^{i}\right) \frac{\partial}{\partial q^{i}}+\sum_{j=0}^{r-1} \alpha_{r-1}^{*}\left(w_{j}\right) \\
\quad \times \frac{\partial}{\partial p_{j}}+\alpha_{r-1}^{*}\left(w_{r}\right) \frac{\partial}{\partial p_{r}} .
\end{aligned}
$$

Comparing these two relations it is clear that if one assumes the $(2 k-r+1)$ th-order condition for $K_{r-1}$, which implies $\bar{v}^{2 k-1}{ }^{r}=q^{2 k-r}$, all the coefficients of $K_{r-1}$ are determined, and the additional identity must hold:

$$
\alpha_{r-1}^{*}\left(w_{r}\right)=\sum_{i=0}^{2 k-1-r} q^{i+1} \alpha_{r-1}^{*}\left(\frac{\partial \hat{p}_{r}}{\partial q^{i}}\right) .
$$

But since $w_{r}=\partial L / \partial q^{r}-p_{r-1}$, we have $\alpha_{r-1}^{*}\left(w_{r}\right)=\partial L / \partial q^{r}-\hat{p}_{r-1} ;$ therefore this identity follows from (3.5).

As an immediate result from the preceding proposition, we can show that the following holds true. have:

Proposition 4: For $1 \leqslant r \leqslant k-1$ and any $f \in C^{\infty}\left(P_{r+1}\right)$, we

$\alpha_{r-1}^{*}\left(K_{r} \cdot f\right)=K_{r-1} \cdot \alpha_{r}^{*}(f)$.

We omit the proof, which is straightforward. 


\section{THE PRIMARY CONSTRAINTS IN THE HAMILTONIAN FORMALISM}

Neither the energy $E_{r}$ nor the evolution operators $K_{r}$ can be constructed in phase space $P_{k}$. But it has a useful structure: its canonical two-form $\omega_{k}$, which is symplectic. So it defines a vector $P_{k}$-bundle isomorphism $\Omega_{k}: T\left(P_{k}\right) \rightarrow T^{*}\left(P_{k}\right)$, and the corresponding isomorphism between the spaces of sections, namely vector fields and differential one-forms. In particular, for each function $f$ in $P_{k}$ a vector field can be constructed:

$$
X_{f}:=\Omega_{k}^{-1} \circ d f
$$

with local expression

$$
X_{f}=\sum_{i=0}^{k-1}\left(\frac{\partial f}{\partial p_{i}} \frac{\partial}{\partial q^{i}}-\frac{\partial f}{\partial q^{i}} \frac{\partial}{\partial p_{i}}\right)
$$

The Poisson bracket of two functions in $P_{k}$ is also defined in the usual way.

From now on it is assumed that the partial Ostrograds$k i$ 's transformations have constant rank $2 k n-m$. Then, locally, the image $P_{r+1}^{(1)}$ of $\alpha_{r}$ can be assumed to be a closed submanifold of $P_{r+1}$ of codimension $m$, defined by the vanishing of $m$ functions $\phi_{r+1}^{\mu}$ in $P_{r+1}$, the primary constraints, whose differentials are linearly independent at each point of $P(1)$.

The functions vanishing on $P_{r+1}^{(1)}$ are also called primary constraints, and constitute an ideal of $C^{\infty}\left(P_{r+1}\right)$ generated by the $\phi_{r+1}^{\mu}$. This is also the set of functions vanishing through the pull-back of $\alpha_{r}$. The $C^{\infty}\left(P_{r+1}\right)$-module of oneforms along $\alpha_{r}$ vanishing through the pull-back of $\alpha_{r}$ is therefore generated by the $d \phi_{r+1}^{\mu}{ }^{\circ} \alpha_{r}$.

Accordingly, we will call primary Hamiltonian constraints the constraints locally defining the submanifold $\alpha_{k-1}\left(P_{k-1}\right)=P_{k}^{(1)}$ of $P_{k}$. Although one could apply this name to the constraints defining the image of $F L$, our terminology is usual in the literature, since these constraints arise form the singularity of the Lagrangian, and particularly from the definition of the momenta $p_{k-1}$ (see for instance Ref. 31, for the second-order case). We shall see also that these primary Hamiltonian constraints are enough to generate all the Hamiltonian constraints through the Dirac's stabilization algorithm, so they play the same role as the primary Hamiltonian constraints for first-order Lagrangians. An additional reason is given by Proposition 9.

Since the primary Hamiltonian constraints arise from the "definition" of $p_{k-1}\left(q^{0}, \ldots, q^{k} ; p_{0}, \ldots, p_{k-2}\right)$ $=\hat{p}_{k-1}\left(q^{0}, \ldots, q^{k}\right)$, the $\phi_{k}^{\mu}$ can be chosen so that they do not depend on $p_{0}, \ldots, p_{k-2}$. This result is shown in Refs. 31 and 36. An intrinsic proof is also available, ${ }^{55}$ provided that this nondependence is given a geometric expression. We comment briefly on this point.

$P_{k}$ is fibered in several ways. The more obvious one, compounding $\gamma_{k}$ with the projections to lower-order tangent bundles:

$$
\begin{aligned}
& \omega^{l}: P_{k} \rightarrow T^{l} Q \quad(0 \leqslant l \leqslant k-1), \\
& \left(q^{0}, \ldots, p_{k-1}\right) \mapsto\left(q^{0}, \ldots, q^{l}\right) .
\end{aligned}
$$

On the other hand it is known the vector $T^{k-1} Q$-bundle epimorphism (2.3)

$$
\begin{aligned}
& \varpi_{l}: P_{k} \rightarrow V_{l}\left(T^{k-1} Q\right)^{*} \quad(k-1 \geqslant l \geqslant 0), \\
& \left(q^{0}, \ldots, p_{k-1}\right) \mapsto\left(q^{0}, \ldots, q^{k-1}, p_{l+1}, \ldots, p_{k-1}\right) .
\end{aligned}
$$

With this, the following vector subbundles of $T\left(P_{k}\right)$ can be constructed:

$$
\text { Ker } T\left(\varpi_{l}\right)=\left\langle\frac{\partial}{\partial p_{0}}, \ldots, \frac{\partial}{\partial p_{l}}\right\rangle \text {. }
$$

Taking $l=k-2$, it is clear from the result stated before that we have the following.

Lemma 1: The primary Hamiltonian constraints $\phi_{k}^{\mu}$ can be chosen to be $\varpi_{k-2}$ projectable-i.e., not depending on $p_{0}, \ldots, p_{k-2}$.

From now on this choice is assumed. This will make the computations much easier.

Finally we want to notice that there is another way to say that a function $f$ in $P_{k}$ does not depend on $p_{0}, \ldots, p_{l}$. To this end compute

$$
T\left(o_{l}^{k-1}\right) \circ T\left(\gamma_{k}\right) \circ X_{f}=\sum_{i=0}^{l} \frac{\partial f}{\partial p_{i}} \frac{\partial}{\partial q^{i}} .
$$

Therefore, $f$ is $\widetilde{\omega}_{l}$ projectable if and only if the above expression is zero.

In particular we have, for the primary constraints,

$$
T\left(o_{k-2}^{k-1}\right) \circ T\left(\gamma_{k}\right) \circ X_{\phi_{k}^{\mu}}=0,
$$

provided that they are chosen as in the lemma.

\section{THE KERNELS OF THE PARTIAL OSTROGRADSKII’'S TRANSFORMATIONS}

Since $\operatorname{Ker} T\left(\alpha_{r}\right) \subset T\left(P_{r}\right)$ is a vector subbundle of rank $m(0 \leqslant r \leqslant k-1), P_{r}$ can be covered by open sets on which $\operatorname{Ker} T\left(\alpha_{r}\right)$ has a frame given by $m$ vector fields $\Gamma_{\mu}^{r}$. It is interesting to have explicit expressions for these vector fields, in order to compute the projectability of functions through the partial Ostrogradskilı's transformations.

We put for convenience

$\Gamma_{\mu}^{k}:=X_{\phi_{k}^{\mu}}$.

Using the preceding lemma, these vector fields have local expression

$$
\Gamma_{\mu}^{k}=\frac{\partial \phi_{k}^{\mu}}{\partial p_{k-1}} \frac{\partial}{\partial q^{k-1}}-\sum_{i=0}^{k-1} \frac{\partial \phi_{k}^{\mu}}{\partial q^{i}} \frac{\partial}{\partial p_{i}} .
$$

Then the $m$ vector functions

$$
\gamma_{\mu}:=\alpha_{k-1}^{*}\left(\frac{\partial \phi_{k}^{\mu}}{\partial p_{k-1}}\right)
$$

are a basis for Ker $W$, since $W \gamma_{\mu}=\partial\left(\alpha_{k-1}^{*}\left(\phi_{k}^{\mu}\right)\right) / \partial q^{k}=0$, and are linearly independent; the reason is that the $\partial \phi_{k}^{\mu} / \partial p_{k-1}$ are already independent, since the constraintsthe image of $\alpha_{k-1}$-appear by the definition of $p_{k-1}$.

Notice also the dependence $\gamma_{\mu}\left(q^{0}, \ldots, q^{k}\right)$, due to the dependences $\phi_{k}^{\mu}\left(q^{0}, \ldots, q^{k-1} ; p_{k-1}\right)$ and $\hat{p}_{k-1}\left(q^{0}, \ldots, q^{k}\right)$.

Proposition 5: The vector bundle Ker $T\left(\alpha_{r}\right)$ admits as a local frame $m$ vector fields $\Gamma_{\mu}^{r}$ with local expression

$$
\Gamma_{\mu}^{r}=\gamma_{\mu} \frac{\partial}{\partial q^{2 k-1-r}},
$$


with $\gamma_{\mu}$ defined by (9.3).

A quick inspection of the Jacobian matrix of $\alpha_{r}$ shows that these $\Gamma_{\mu}^{r}$, which are linearly independent, constitute a frame for $\operatorname{Ker} T\left(\alpha_{r}\right)$. Then it is easily checked that they transform as vector fields.

An intrinsic construction of these vector fields can also be provided using the vertical lift of the higher-order tangent bundles. ${ }^{55}$

Now we can easily test the local projectability of a function $f \in C^{\infty}\left(P_{r}\right)$ through $\alpha_{r}: \Gamma_{\mu}^{r} \cdot f=0$ for all $\mu$. If $\alpha_{r}$ is moreover assumed to have connected fibers and $\alpha_{r}\left(P_{r}\right) \subset P_{r+1}$ to be a closed submanifold this also ensures the global $\alpha_{r}$ projectability.

It is also known that the vector subbundles Ker $T\left(\alpha_{r}\right) \subset T\left(P_{r}\right)$ are completely integrable. We have indeed

$$
\left[\Gamma_{\mu}^{r}, \Gamma_{\nu}^{r}\right]=0, \quad 0 \leqslant r \leqslant k-1,
$$

which follows from an elementary calculation involving $\Gamma_{\mu}^{r} \cdot \gamma_{v}=0$. This property is not always true for the $\Gamma_{\mu}^{k}$ since

$$
\left[\Gamma_{\mu}^{k}, \Gamma_{v}^{k}\right]=-X_{\left\{\phi_{k}^{k}, \phi_{k}^{k}\right\}} .
$$

We finish by proving an interesting commutation relation between the intermediate evolution operators and the vector fields presently constructed.

Proposition 6: For $0 \leqslant r \leqslant k-1$ and any function $g \in C^{\infty}\left(P_{r+1}\right)$,

$$
\Gamma_{\mu}^{r} \cdot\left(K_{r} \cdot g\right)=\alpha_{r}^{*}\left(\Gamma_{\mu}^{r+1} \cdot g\right)
$$

We take the local expression

$$
\begin{aligned}
K_{r} \cdot g= & \alpha_{r}^{*}\left(\frac{\partial g}{\partial q^{0}}\right) q^{1}+\cdots+\alpha_{r}^{*}\left(\frac{\partial g}{\partial q^{2 k-2-r}}\right) q^{2 k-1-r} \\
& +\alpha_{r}^{*}\left(\frac{\partial g}{\partial p_{0}}\right)\left(\frac{\partial L}{\partial q^{0}}\right)+\cdots \\
& +\alpha_{r}^{*}\left(\frac{\partial g}{\partial p_{r}}\right)\left(\frac{\partial L}{\partial q^{r}}-p_{r-1}\right)
\end{aligned}
$$

and apply $\Gamma_{\mu}^{r}=\gamma_{\mu} \partial / \partial q^{2 k-1-r}$ to it. This yields

$$
\begin{aligned}
\Gamma_{\mu}^{r} \cdot\left(K_{r} \cdot g\right)= & \left(\alpha_{r}^{*}\left(\frac{\partial g}{\partial q^{2 k-2-r}}\right)\right. \\
& \left.+\sum_{i=0}^{r} \alpha_{r}^{*}\left(\frac{\partial g}{\partial p_{i}}\right) \frac{\partial^{2} L}{\partial q^{i} \partial q^{2 k-1-r}}\right) \gamma_{\mu},
\end{aligned}
$$

where the summation only contributes when $r=k-1$.

On the other hand, for $r \neq k-1$ it is clear that

$$
\alpha_{r}^{*}\left(\Gamma_{\mu}^{r+1} \cdot g\right)=\alpha_{r}^{*}\left(\frac{\partial g}{\partial q^{2 k-2-r}}\right) \gamma_{\mu},
$$

which is the same as (9.7). If $r=k-1$ then

$$
\begin{aligned}
\alpha_{k-1}^{*}\left(\Gamma_{\mu}^{k} \cdot g\right)= & \alpha_{k-1}^{*}\left(\frac{\partial g}{\partial q^{k-1}}\right) \gamma_{\mu} \\
& -\sum_{i=0}^{k-1} \alpha_{k-1}^{*}\left(\frac{\partial g}{\partial p_{i}}\right) \alpha_{k-1}^{*}\left(\frac{\partial \phi_{k}^{\mu}}{\partial q^{i}}\right) .
\end{aligned}
$$

It suffices to prove

$$
\begin{aligned}
\alpha_{k-1}^{*}\left(\frac{\partial \phi_{k}^{\mu}}{\partial q^{i}}\right) & =-\frac{\partial \hat{p}_{k-1}}{\partial q^{i}} \alpha_{k-1}^{*}\left(\frac{\partial \phi_{k}^{\mu}}{\partial p_{k-1}}\right) \\
& =-\frac{\partial^{2} L}{\partial q^{i} \partial q^{k}} \gamma_{\mu},
\end{aligned}
$$

which follows from chain's rule and the fact that $\phi_{k}^{\mu}$ is a primary constraint.

As an application, we have the following.

Corollary: $K_{r} \cdot g$ is an $\alpha_{r}$-projectable function if and only if, for all $\mu, \Gamma_{\mu}^{r+1} \cdot g$ is a primary constraint in $P_{r+1}$.

\section{EQUATIONS OF MOTION FOR PATHS IN THE INTERMEDIATE SPACES $\boldsymbol{P}_{0}, \ldots, \boldsymbol{P}_{k-1}$}

We consider an $n$-dimensional differentiable manifold $Q$ (configuration space) and a $k$ th-order Lagrangian $L \in C^{\infty}\left(T^{k} Q\right)$. For a curve in $Q$ the Euler-Lagrange equations read $[L]_{q(r)}=0$, with

$$
[L]=\sum_{i=0}^{k}(-1)^{i} d_{T}^{l}\left(\frac{\partial L}{\partial q^{i}}\right)=\frac{\partial L}{\partial q^{0}}-d_{r} \hat{p}_{0} .
$$

This system of $n$ equations has order $\leqslant 2 k$; its order is $2 k$ if and only if the Hessian matrix $W$ of $L$ does not vanish identically.

The same motion can be described by first-order equations, but these should be established on a suitable manifold fibered over $Q$. It is usually done in $T^{2 k-1} Q$, but also the Hamiltonian formalism in $T^{*}\left(T^{k-1} Q\right)$ is available. In fact, we are going to write equivalent equations of motion in every intermediate space $P_{r}(0 \leqslant r \leqslant k)$; however, the Hamiltonian formalism (that of $P_{k}$ ) is delayed to another section.

Let $\xi$ be a path in $Q$. Its $(2 k-1)$ th derivative is its natural lift $\xi_{0}$ to $P_{0} \cong T^{2 k-1} Q$. Then, by composition with the partial Ostrogradskiî's transformations one gets in a very natural way paths $\xi_{r}$ in all the intermediate spaces $P_{r}$. To abbreviate, we say that the paths constructed in this way are equivalent.

We now look for a first-order differential equation for a path in $P_{r}$; this path should be equivalent to another one in $Q$, solution of the Euler-Lagrange equations. In Ref, 46 the equations of motion for a first-order Lagrangian are written as

$$
T(F L) \circ \dot{\xi}=K \circ \xi,
$$

where $K$ is defined by Eqs. (6.2)-(6.4). Now we shall proceed in a similar way.

Theorem 5: The equation of motion in $P_{r}$ for a path $\xi_{r}$ $(0 \leqslant r \leqslant k-1)$ is

$$
T\left(\alpha_{r}\right) \circ \dot{\xi}_{r}=K_{r} \circ \xi_{r} .
$$

This equation holds if and only if $\xi_{r}$ is equivalent to a path $\xi$ in $Q$ which is a solution of the Euler-Lagrange equations. It also holds if and only if for every $g \in C^{\infty}\left(P_{r+1}\right)$

$$
\frac{d}{d t}\left(\alpha_{r}^{*}(g) \circ \xi_{r}\right)=\left(K_{r} \cdot g\right) \circ \xi_{r} .
$$

This is illustrated by the following diagram: 


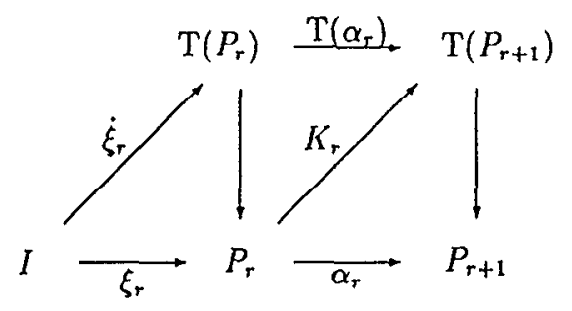

What is really easy to prove is that the equations of motion for $\xi_{r}$ in $P_{r}$ imply those of $\xi_{r+1}=\alpha_{r} \circ \xi_{r}$ in $P_{r+1}$ $(0 \leqslant r \leqslant k-2)$, since

$$
\begin{aligned}
T\left(\alpha_{r+1}\right) \circ \dot{\xi}_{r+1} & =T\left(\alpha_{r+1}\right) \circ T\left(\alpha_{r}\right) \circ \dot{\xi}_{r} \\
& =T\left(\alpha_{r+1}\right) \circ K_{r} \circ \xi_{r} \\
& =K_{r+1} \circ \alpha_{r} \circ \xi_{r} \\
& =K_{r+1} \circ \xi_{r+1}
\end{aligned}
$$

where relation (7.1) has been used.

The last statement of the theorem is also straightforward, since

$$
\begin{aligned}
\frac{d}{d t}\left(\alpha_{r}^{*}(g) \circ \xi_{r}\right) & =\left\langle d \alpha_{r}^{*}(g) \circ \xi_{r}, \dot{\xi}_{r}\right\rangle \\
& =\left\langle{ }^{i} T_{\xi_{r}}\left(\alpha_{r}\right) \cdot\left(d g \circ \alpha_{r} \circ \xi_{r}\right), \dot{\xi}_{r}\right\rangle \\
& =\left\langle d g \circ \alpha_{r} \circ \xi_{r}, T\left(\alpha_{r}\right) \circ \dot{\xi}_{r}\right\rangle \\
& =\left\langle d g \circ \alpha_{r} \circ \xi_{r}, K_{r} \circ \xi_{r}\right\rangle \\
& =\left(K_{r} \circ g \circ \circ \xi_{r},\right.
\end{aligned}
$$

and conversely the value of $T\left(\alpha_{r}\right) \circ \dot{\xi}_{r}$ is determined by its contraction with the exact one-forms $d g$ in $P_{r+1}$, along the path $\alpha_{r} \circ \xi_{r}$.

Now let us prove the equivalence with the Euler-Lagrange equations. First consider $P_{0}$ and a curve $\xi_{0}$ therein, with local expression $\left(q^{0}, \ldots, q^{2 k-1}\right)$. If it is the natural lift of a solution $\xi$ in $Q$ with local expression $q=q^{0}$ then we must have $d q^{i} / d t=q^{i+1}$ for $0 \leqslant i \leqslant 2 k-2$, and $d \hat{p}_{0} / d t=\partial L / \partial q^{0}$ -this comes from the Euler-Lagrange equations (10.1). Conversely, a curve in $P_{0}$ satisfying all these conditions is clearly the lift to $P_{0}$ of a solution in $Q$, with local expression $q=q^{0}$.

Next take $P_{1}$ and a curve $\xi_{1}$ with local expression $\left(q^{0}, \ldots, q^{2 k-2} ; p_{0}\right)$. If it comes from a solution in $Q$ then we must have $d q^{i} / d t=q^{i+1}$ for $0 \leqslant i \leqslant 2 k-3, \quad p_{0}$ $=\hat{p}_{0}\left(q, d q / d t, \ldots, d^{2 k-1} q / d t^{2 k-1}\right)$ and $d \hat{p}_{0} / d t=\partial L / \partial q^{0}$. Of these three conditions the third one can be changed to $d p_{0} / d t=\partial L / \partial q^{0}$. The second one can be, in view of the relation between $\hat{p}_{0}$ and $\hat{p}_{1}(3.5)$, and the properties of $d_{T}$, equivalently written $d \hat{p}_{1} / d t=\partial L / \partial q^{1}-p_{0}$. Conversely, a curve in $P_{1}$ satisfying these conditions is obtained from a solution $\xi$ in $Q$, with local expression $q=q^{0}$, since

$$
\frac{\partial L}{\partial q^{0}}-\frac{d}{d t} \hat{p}_{0}\left(q, \frac{d q}{d t}, \ldots, \frac{d^{2 k-1} q}{d t^{2 k-1}}\right)=\frac{\partial L}{\partial q^{0}}-\frac{d p_{0}}{d t}=0 .
$$

The same argument works in every intermediate space $P_{r}$, for $0 \leqslant r \leqslant k-1$. A curve $\xi_{r}$ therein with local expression $\left(q^{0}, \ldots, q^{2 k-1-r_{;}} p_{0}, \ldots, p_{r-1}\right)$ is equivalent to a solution $\xi$ in the base $Q$ with local expression $q=q^{0}$ of the Euler-Lagrange equations if and only if it satisfies

$$
\begin{aligned}
& \frac{d q^{i}}{d t}=q^{i+1}, \quad 0 \leqslant i \leqslant 2 k-2-r, \\
& \frac{d p_{j}}{d t}=\frac{\partial L}{\partial q^{j}}-p_{j-1}, \quad 0 \leqslant j \leqslant r-1, \\
& \frac{d \hat{p}_{r}}{d t}=\frac{\partial L}{\partial q^{r}}-p_{r-1},
\end{aligned}
$$

where it is understood that $p_{-1}=0$.

Now let us compute the local expression of the equations of motion (10.2):

$$
\left(\begin{array}{ccccccc}
I_{n} & & & & 0_{n} & 0_{n} & \cdots \\
& \ddots & & & \vdots & \vdots & \\
& & \ddots & & \vdots & \vdots & \\
& & & I_{n} & 0_{n} & 0_{n} & \cdots \\
0_{n} & \cdots & \cdots & 0_{n} & 0_{n} & I_{n} & \\
\vdots & & & \vdots & \vdots & & \ddots \\
0_{n} & \cdots & \cdots & 0_{n} & 0_{n} & & \\
\partial \hat{p}_{r} / \partial q^{0} & \cdots & \cdots & \cdots & \partial \hat{p}_{r} / \partial q^{2 k-1-r} & 0_{n} & \cdots
\end{array}\right.
$$

These $2 k n$ equations are the same as (10.4), and this finishes the proof.

Equation (10.3) illustrates the meaning of $K_{r}$ : it is a time-evolution operator, conveniently expressed in order to avoid the ambiguity underlying constrained systems. Similarly the following vector field along $F L$ can be considered:

$$
K:=K_{k-1} \circ \alpha_{k-2} \circ \cdots \circ \alpha_{0}=T\left(\alpha_{k-1} \circ \cdots \circ \alpha_{1}\right) \circ K_{0}
$$

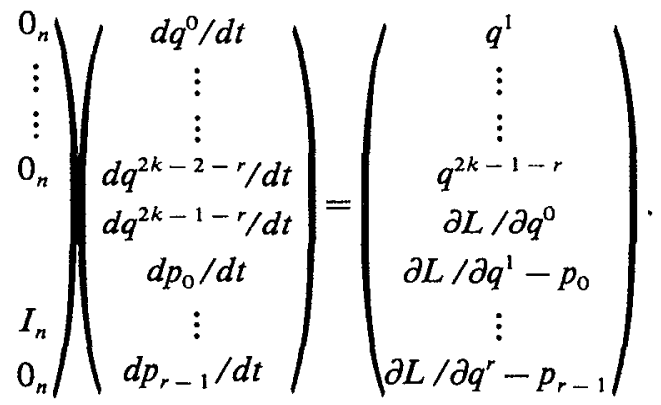

(it is the same, according to Proposition 3). Then, a consequence of Theorem 1 is that

$$
\frac{d}{d t}\left(F L *(g) \circ \xi_{0}\right)=(K \cdot g) \circ \xi_{0} .
$$

The problem posed by singular Lagrangians can be realized by looking at the equations of motion. In the coordinate version, the evolution of $q^{2 k-1-r}$ is hidden in Eq. (10.4c). 
Using the definition of $\hat{p}_{r}$ this equation can be rewritten

$$
\begin{aligned}
W \frac{d q^{2 k-1-r}}{d t}= & (-1)^{k-1-r} \\
& \times\left(\frac{\partial L}{\partial q^{r}}-p_{r-1}-\sum_{i=0}^{2 k-2-r} \frac{\partial \hat{p}_{r}}{\partial q^{i}} q^{i+1}\right) .
\end{aligned}
$$

If $L$ is singular, $W$ is not invertible and this equation cannot be written in normal form, thus the existence and uniqueness theorem for differential equations cannot be applied.

In the geometric version, if $\alpha_{r}$ is a diffeomorphism then the equation of motion can be written

$$
\dot{\xi}_{r}=T\left(\alpha_{r}\right)^{-1} \circ K_{r} \circ \xi,
$$

and for every initial condition in $P_{r}$ there passes an integral curve of the vector field $T\left(\alpha_{r}\right)^{-1}{ }^{1} K_{r}$. But if $L$ is singular then $T\left(\alpha_{r}\right)$ cannot be locally inverted and the evolution of $\xi_{r}$ is not determined by a vector field in $P_{r}$. This is discussed in the following section.

\section{EQUATIONS OF MOTION FOR VECTOR FIELDS IN THE INTERMEDIATE SPACES $\boldsymbol{P}_{0}, \ldots, \boldsymbol{P}_{k-1}$}

It is usual in the literature to shift the problem of finding the solutions of the equations of motion into the problem of obtaining the vector fields $X_{r}$ whose integral curves are these solutions. If $L$ is singular it can be expected that such vector fields can only be properly defined on a submanifold of $P_{r}$ covered by solutions of the equations of motion, and they will not be uniquely defined, for a given set of initial conditions.

In view of the last section, a relation between $X_{r}$ and $K_{r}$ is expected. On the other hand the dynamical fields of a firstorder Lagrangian formalism satisfy a "presymplectic equation" and the "second-order condition." "Both features are contained in the following theorem.

Theorem 6: Suppose that $S$ is a closed submanifold of $P_{\text {r }}$ $(0 \leqslant r \leqslant k-1)$ such that there are solutions of the equations of motion passing by every point of $S$. Let $X_{r}$ be a vector field in $P_{r}$ tangent to $S$. Then the integral curves of $X_{r}$ passing by $S$ are solutions of the equations of motion in $P_{r}$ if and only if $X_{r}$

satisfies the following equation [The notation $=$ means equality on the submanifold $S$ (weak equality) ]:

$$
T\left(\alpha_{r}\right) \circ X_{r} \underset{S}{=} K_{r} .
$$

Moreover, this equation can be equivalently written as the two following equations: the presymplectic equation

$$
i_{X_{r}} \omega_{r} \underset{s}{=} d E_{r}
$$

and the $(2 k-r)$ th-order condition

$$
T\left(o_{2 k-2-r-r}^{2 k-1-r}\right) \circ T\left(\gamma_{r}\right) \circ X_{r}=\underset{s}{=} j^{2 k-2-r^{\circ}} \gamma_{r} .
$$

For $x \in S$ let $\xi_{r}$ be an integral curve of $X_{r}$ passing by $x$, and suppose that $\xi_{r}$ is a solution of the equations of motion in $P_{r}$. Then, $T\left(\alpha_{r}\right) \circ X_{r} \circ \xi_{r}=T\left(\alpha_{r}\right) \circ \xi_{r}=K_{r} \circ \xi_{r}$, which proves equality $(11.1)$ at the point $x$. Conversely, assume (11.1) and let $\xi_{r}$ be an integral curve of $X$, passing by $S$.
Since $X_{r}$ is tangent to $S$, all the image of $\xi_{r}$ is contained in $S$, and $T\left(\alpha_{r}\right) \circ \xi_{r}=T\left(\alpha_{r}\right) \circ X_{r} \circ \xi_{r}=K_{r} \circ \xi_{r}$, which gives the equation of motion for $\xi_{r}$.

In order to prove the equivalence between (11.1) and $(11.2),(11.3)$ the intrinsic definition of $K_{r}$ can be used. First,

$$
\begin{aligned}
i_{X_{r}} \omega_{r} & =i_{X_{r}} \alpha_{r}^{*}\left(\omega_{r+1}\right) \\
& =\alpha_{r}^{*}\left(i_{T\left(\alpha_{r}\right) \otimes X_{r}} \omega_{r+1}\right) \\
& =\alpha_{r}^{*}\left(i_{K_{r}} \omega_{r+1}\right) \\
& =d E_{r},
\end{aligned}
$$

which proves $(11.2)$. On the other hand, since we have $o_{2 k-2-r}^{2 k-1-r} \circ \gamma_{r}=\gamma_{r+1} \circ \alpha_{r}$,

$$
\begin{aligned}
T\left(o_{2 k-2-r}^{2 k-1-r}\right) \circ T\left(\gamma_{r}\right) \circ X_{r} & =T\left(\gamma_{r+1}\right) \circ T\left(\alpha_{r}\right) \circ X_{r} \\
& =T\left(\gamma_{r+1}\right) \circ K_{r} \\
& =j^{2 k-2-r^{2} \circ \gamma_{r},}
\end{aligned}
$$

which proves (11.3).

Conversely, let $X$, satisfy these relations and notice that actually the characterization of $K_{r}$ remains valid for a vector field along $\alpha$, considered to be defined only on a submanifold $S \subseteq P_{r}$. But a vector feld such as $T\left(\alpha_{r}\right) \circ X_{r}$ satisfies the conditions needed, since

$$
\begin{aligned}
& T\left(\gamma_{r+1}\right) \circ\left(T\left(\alpha_{r}\right) \circ X_{r}\right)=T\left(o_{2 k-2-r}^{2 k-1-r}\right) \circ T\left(\gamma_{r}\right) \circ X_{r} \\
& =j^{2 k-2-r^{\circ} \circ \gamma_{r},} \\
& \begin{aligned}
\alpha_{r}^{*}\left(i_{T\left(\alpha_{r}\right) X_{r}} \omega_{r+1}\right)= & i_{X_{r}} \alpha_{r}^{*}\left(\omega_{r+1}\right) \\
= & i_{X_{r}} \omega_{r} \\
& =d E_{r} .
\end{aligned}
\end{aligned}
$$

This completes the proof.

If the local expression of $X_{r}$ is

$$
X_{r}=\sum_{i=0}^{2 k-1-r} v^{i} \frac{\partial}{\partial q^{i}}+\sum_{j=0}^{r-1} w_{j} \frac{\partial}{\partial p_{j}}
$$

then these equations of motion for $X_{r}$ read, from (10.4) and (10.7),

$$
\begin{aligned}
& v_{s}^{i}=q^{i+1}, \quad 0 \leqslant i \leqslant 2 k-2-r \\
& w_{j}=\frac{\partial L}{\partial q^{i}}-p_{j-1}, \quad 0 \leqslant j \leqslant r-1, \\
& W v^{2 k-1-r} \\
& \quad=(-1)^{k-1-r}\left(\frac{\partial L}{\partial q^{r}}-p_{r-1}-\sum_{i=0}^{2 k-2-r} \frac{\partial \hat{p}_{r}}{\partial q^{i}} q^{i+1}\right) .
\end{aligned}
$$

For a regular Lagrangian, Theorem 2 has a simple expression since $\Omega_{r}$ is a vector bundle isomorphism.

Proposition 7: Suppose that $L$ is a regular Lagrangian. Then the relation $i_{X_{r}}=\omega_{r}=d E_{r}$ has one and only one solution,

$$
X_{r}=\Omega_{r}^{-1} \circ d E_{r}
$$


which also satisfies the $(2 k-r)$ th-order condition.

The first statement is obvious. To prove the second one it suffices to show that $T\left(\alpha_{r}\right) \circ X_{r}=K_{r}$. But for a regular Lagrangian $K_{r}$ can be characterized by the only property of $\alpha_{r}^{*}\left(i_{k r} \omega_{r+1}\right)=d E_{r}$ (see Proposition 2) and this has been proven for $K_{r}=T\left(\alpha_{r}\right) \circ X_{r}$ in the preceding theorem under the only hypothesis currently considered.

Of course, this result was already known in the Lagrangian formalism. ${ }^{29}$ If $L$ is singular then the $(2 k-r)$ th-order condition must be explicitly imposed to obtain the correct dynamics. However, one can also discuss other "dynamics" which include a nonmaximal order condition. ${ }^{57}$

\section{EQUATIONS OF MOTION IN THE HAMILTONIAN FORMALISM}

The energy function of $P_{k-1}, E_{k-1}$, is (locally) $\alpha_{k-1}$ projectable: if the $m$ vector fields $\Gamma_{\mu}^{k-1}=\gamma_{\mu} \partial / \partial q^{k}$, which are a frame for $\operatorname{Ker} T\left(\alpha_{r}\right)$, are applied to $p_{0} q^{l}+\ldots+p_{k-2} q^{k-1}+\hat{p}_{k-1} q^{k}-L\left(q^{0}, \ldots, q^{k}\right)$ the result is zero.

A Hamiltonian function is any $\mathrm{H \in C} C^{\infty}\left(P_{k}\right)$ such that $\alpha_{k-1}^{*}(H)=E_{k-1}$. Such functions exist locally, but for simplicity we can assume them to be defined everywhere. Two Hamiltonians differ from a function vanishing on the primary constraint submanifold $P_{k}^{(1)}$, that is to say, a $C^{\infty}\left(P_{k}\right)$ linear combination of the primary constraints $\phi_{k}^{\mu}$. However, like the primary constraints, a special class of Hamiltonians will be selected, in order to make computations easier. ${ }^{33}$

When studying the projectability of $E_{k-1}$, the term $\Sigma_{i=0}^{k-2} p_{i} q^{i+1}$ plays no role, and is clearly projectable. The term $\hat{p}_{k-1} q^{k}-L$ only depends on $q^{0}, \ldots, q^{k}$, therefore it can be projected to a function $h\left(q^{0}, \ldots, q^{k-1}, p_{k-1}\right)$. We can write a Hamiltonian

$$
H=\sum_{i=0}^{k-2} p_{i} q^{i+1}+h\left(q^{0}, \ldots, q^{k-1} ; p_{k-1}\right) .
$$

Although the term $\Sigma_{i=0}^{k-2} p_{i} q^{i+1}$ is not coordinate invariant, this particular expression can be given a geometric meaning. ${ }^{55}$ Indeed, our particular choice of $H$ is intrinsically expressed by

$$
T\left(o_{k-2}^{k-1}\right) \circ T\left(\gamma_{k}\right) \circ X_{H}=j^{k-2} \circ \gamma_{k},
$$

which is another way to express that $\partial H / \partial p_{i}=q^{i+1}$ for $0<i \leqslant k-2$. For obvious reasons it can be called the $k t h$ order condition for $X_{H}$.

Now we are ready to look for the equation of motion in $P_{k}$. A coordinate analysis can be performed as in (10.4). The result is

$$
\begin{aligned}
& \frac{d q^{i}}{d t}=q_{s}^{i+1}, \quad 0 \leqslant i \leqslant k-2, \\
& \frac{d p_{j}}{d t}=\frac{\partial L}{\partial q^{j}}-p_{j-1}, \quad 0 \leqslant j \leqslant k-1, \\
& p_{k-1}=\hat{p}_{s-1}=\frac{\partial L}{\partial q^{k}},
\end{aligned}
$$

where it is understood that $p_{-1}=0$ and that the fuctions $\partial L / \partial q^{i}$ are computed along the motion using $q^{k}=d q^{k-1} / d t$.
However, we want to develop a Hamiltonian formalism in $P_{k}$. Let $\xi_{k}$ be a path therein. If it is equivalent to a solution $\xi_{k-1}$ in $P_{k-1}$ then it obeys $\dot{\xi}_{k}=\left(\alpha_{k-1} \circ \xi_{k-1}\right)=T\left(\alpha_{k-1}\right) \circ \dot{\xi}_{k-1}=K_{k-1} \circ \xi_{k-1}$.

Conversely, suppose we have $\xi_{k}$ in $P_{k}^{(1)}$ and that $\dot{\xi}_{k}=K_{k-1} \circ \xi_{k-1}$, where $\xi_{k-1}$ is any path in $P_{k-1}$ such that $\xi_{k}=\alpha_{k-1} \circ \xi_{k-1}$. Then it follows that $\xi_{k-1}$ is a solution of the equations of motion, therefore $\xi_{k}$ is equivalent to a solution of the Euler-Lagrange equations.

We would like to get rid of $\xi_{k-1}$ in the equations of motion in $P_{k}$. To this end $K_{k-1}$ should be related to $X_{H}$. By definition $\alpha_{k-1}^{*}\left(i_{K_{k-1}} \omega_{k}\right)=d E_{k-1}=d \alpha_{k-1}^{*}(H)$, therefore

$$
\alpha_{k-1}^{*}\left(i_{K_{k-1}} \omega_{k}-d H \circ \alpha_{k-1}\right)=0 .
$$

Since the one-forms along $\alpha_{k-1}$ admit the $d \phi_{k}^{\mu} \circ \alpha_{k-1}$ as a (local) basis, there exist $m$ functions $\lambda_{k-1}^{\mu}$, uniquely defined by the choice of $H$ and $\phi_{k}^{\mu}$, such that

$$
i_{K_{k-1}} \omega_{k}-d H^{\circ} \alpha_{k-1}=\sum_{\mu} \lambda_{k-1}^{\mu} d \phi_{k}^{\mu} \circ \alpha_{k-1} .
$$

Using $\Omega_{k}^{-1}$ this relation yields the following.

Proposition 8: Let $H$ be any Hamiltonian function and $\phi_{k}^{\mu}$ a set of primary Hamiltonian constraints. Then there exist $m$ functions $\lambda_{k-1}^{\mu}$ uniquely defined in $P_{k-1}$ such that

$$
K_{k-1}=X_{H}^{\circ} \alpha_{k-1}+\sum_{\mu} \lambda_{k-1}^{\mu} X_{\phi_{k}^{\mu}} \circ \alpha_{k-1} .
$$

Moreover, if the constraints satisfy (8.3) and $H$ satisfies the $k$ th-order condition (12.2), then the functions $\lambda_{k-1}^{\mu}$ are $\gamma_{k-1}$ projectable-i.e., do not depend on the momenta.

The last statement can be easily obtained in coordinates from the coefficient of $\partial / \partial q^{2 k-2-r}$.

Thanks to the above proposition, the equation of motion $\dot{\xi}_{k}=K_{k-1} \circ \xi_{k-1}$ can be rewritten

$$
\dot{\xi}_{k}=X_{H} \circ \xi_{k}+\sum_{\mu}\left(\lambda_{k-1}^{\mu} \circ \xi_{k-1}\right)\left(X_{\phi_{k}^{\alpha}}^{\circ} \xi_{k}\right) .
$$

Let $\xi_{k}$ be a path in $P_{k}^{(1)}$ satisfying this relation. We can set $\eta^{\mu}(t)=\lambda_{k-1}^{\mu}\left(\xi_{k-1}(t)\right)$, which are $m$ functions of time. Then $\xi_{k}$ satisfies

$$
\dot{\xi}_{k}=X_{H} \circ \xi_{k}+\sum_{\mu} \eta^{\mu}\left(X_{\phi_{k}^{\prime}} \circ \xi_{k}\right) .
$$

Conversely, if $\xi_{k}$ satisfies this relation for some functions $\eta^{\mu}(t)$, let $\xi_{k-1}$ be the only path in $P_{k-1}$ such that $\xi_{k}=\alpha_{k-1} \circ \xi_{k-1}$ and $j^{k-1} \circ \gamma_{k-1} \circ \xi_{k-1}=T\left(\gamma_{k}\right) \circ \dot{\xi}_{k}$. Then $\dot{q}^{k-1}$ is

$$
\frac{\partial H}{\partial p_{k-1}}+\sum \eta^{\mu} \gamma_{\mu}
$$

and also

$$
q^{k}=\alpha_{k-1}^{*}\left(\frac{\partial H}{\partial p_{k-1}}\right)+\sum \lambda_{k-1}^{\mu} \gamma_{\mu},
$$

which proves that $\eta^{\mu}=\lambda_{k-1}^{\mu}\left(q^{0}, \ldots, q^{k-1}, \dot{q}^{k-1}\right)$-see Ref. 36.

Theorem 7: Let $\xi_{k}$ be a path in $P_{k}$. It is equivalent to a solution $\xi$ in $Q$ of the Euler-Lagrange equations if and only if its range is in $P_{k}^{(1)}$ and there exist $m$ functions of time $\eta^{\mu}$ such 
that the following equation holds:

$$
\dot{\xi}_{k}=X_{H} \circ \xi_{k}+\sum_{\mu} \eta^{\mu}(t)\left(X_{d_{k}^{\prime}} \circ \xi_{k}\right)
$$

Equivalently, for every $f \in C^{\infty}\left(P_{k}\right)$

$$
\frac{d}{d t}\left(f \circ \xi_{k}\right)=\{f, H\} \circ \xi_{k}+\sum_{\mu} \eta^{\mu}\left\{f, \phi_{k}^{\mu}\right\} \circ \xi_{k} .
$$
tion. $^{43,58}$

Equation (12.5) is called the Hamilton-Dirac equa-

\section{POSITION OF THE STABILIZATION PROBLEM}

If the Lagrangian is singular-what is assumed from now on-relation (11.1) is to be considered as an equation both for the dynamical fields $X_{r}$ and the submanifolds $S \subset P_{r}$ where the motion can take place. This problem will be solved in the following sections, but for the time being let us pose the problem. (This can be considered within a more general formalism. $^{59,60}$ )

The equation $T_{x}\left(\alpha_{r}\right) \cdot X_{r}(x)=K_{r}(x)$ for the unknown vector $X_{r}(x)$ has no solution at every $x \in P_{r}$ because $T_{x}\left(\alpha_{r}\right)$ is not subjective. The set of $x \in P_{r}$ such that the compatibility condition

$$
K_{r}(x) \in \operatorname{Im} T_{x}\left(\alpha_{r}\right)
$$

holds is gencrically a submanifold $P_{r}^{(1)} \subset P_{r}(0 \leqslant r \leqslant k-1)$ which will be called the primary constraint submanifold of $P_{r}$. However, the same name has been applied to the image of $\alpha_{r-1}(1 \leqslant r \leqslant k)$. Let us show that both concepts agree for $1 \leqslant r \leqslant k-1$.

First recall that $K_{r}(x) \in \operatorname{Im} T_{x}\left(\alpha_{r}\right)$ if and only if $K_{r}(x)$ is orthogonal to $\operatorname{Ker}^{t} T_{x}\left(\alpha_{r}\right)$. This is spanned by the $d \phi_{r+1}^{\mu}\left(\alpha_{r}(x)\right)(1 \leqslant \mu \leqslant m)$, and therefore the compatibility condition is that the $m$ functions $\bar{\phi}_{r}^{\mu}:=K_{r} \cdot \phi_{r+1}^{\mu}$ vanish. But the commutation relation (7.2) yields

$$
\begin{aligned}
\alpha_{r-1}^{*}\left(\bar{\phi}_{r}^{\mu}\right) & =\alpha_{r-1}^{*}\left(K_{r} \cdot \phi_{r+1}^{\mu}\right) \\
& =K_{r-1} \cdot \alpha_{r}^{*}\left(\phi_{r+1}^{\mu}\right)=0 .
\end{aligned}
$$

Therefore, the $\bar{\phi}_{r}^{\mu}$ are primary constraints in the sense that they vanish on $\alpha_{r-1}\left(P_{r-1}\right)$. If $\phi_{r+1}^{\mu}$ do not depend on $p_{0}, \ldots, p_{r-1}$, then $\bar{\phi}_{r}^{\mu}$ docs not depend on $p_{0}, \ldots, p_{r-2}$, and the local expression of $K_{r}$ allows us to compute

$$
\frac{\partial \bar{\phi}_{r}^{\mu}}{\partial p_{r-1}}=\frac{\partial}{\partial p_{r-1}}\left(K_{r} \cdot \phi_{r+1}^{\mu}\right)=-\alpha_{r}^{*}\left(\frac{\partial \phi_{r+1}^{\mu}}{\partial p_{r}}\right) .
$$

Since the $m$ vectors $\gamma_{\mu}=\alpha_{k-1}^{*}\left(\partial \phi_{k}^{\mu} / \partial p_{k-1}\right)$ are linearly independent at each point, we have the following.

Proposition 9: Let $\phi_{k}^{\mu}$ be $m$ independent primary constraints for $P_{k}^{(1)} \subset P_{k}$, not depending on $p_{0}, \ldots, p_{k-2}$. For $0 \leqslant r \leqslant k-1$ define inductively

$$
\phi_{r}^{\mu}:=K_{r} \cdot \phi_{r+1}^{\mu} \text {. }
$$

Then the $m$ functions $\phi_{r}^{\mu}$ do not depend on $p_{0}, \ldots, p_{r-2}$ and generate the primary constraints arising from the compatibility condition (13.1). For $r \neq 0$ they are linearly independent and define the submanifold $\alpha_{r-1}\left(P_{r-1}\right) \subset P_{r}$.
Their local expressions are

$$
\begin{aligned}
\phi_{r}^{\mu}= & (-1)^{k-1-r} \gamma_{\mu} \cdot\left(\frac{\partial L}{\partial q^{r}}-p_{r-1}\right. \\
& \left.-\sum_{i=0}^{2 k-2-r} \frac{\partial \hat{p}_{r}}{\partial q^{i}} q^{i+1}\right) .
\end{aligned}
$$

It only remains to prove $(13.3)$; it is computed for $r=k-1$, and the proof goes by induction on $r$.

Notice that Eq. (13.3) could have been obtained from the coordinate expression (10.7) and the fact that the $\gamma_{\mu}$ are a basis for $\operatorname{Ker} W$.

Now suppose that $X_{r}$ is a solution of the equation of motion (11.1). The equality $T\left(\alpha_{r}\right) \circ X_{r} \underset{S}{=} K_{r}$ certainly has solutions with $S=P_{r}^{(1)}$, but they are not necessarily tangent to $P_{r}^{(1)}$. This will be achieved only on a submanifold $S=P_{r}^{(F)}$, and the values of $X_{r}$ will be meaningful only there. However, $X_{r}$ can be extended out of $P_{r}^{(F)}$ as we want; using this freedom we extend $X$, in order to satisfy

$$
T\left(\alpha_{r}\right) \circ X_{r} \underset{p_{r}^{(1)}}{=} K_{r} .
$$

To abbreviate, any vector field $X_{r}$ satisfying this equality will be called a primary dynamical field; we extend this convention for $X_{k} \underset{P_{k}^{(n)}}{=} X_{H}$, for any Hamiltonian $H$. Notice that two primary dynamical fields differ, on $P_{r}^{(1)}$, from a linear combination of the fields $\Gamma_{\mu}^{r}$.

An immediate consequence of the definition is the following proposition.

Proposition 10: $X$, is a primary dynamical field in $P_{r}$ $(0 \leqslant r \leqslant k-1)$ if and only if there exist $m$ vector fields $Y_{\mu}^{r}$ along $\alpha_{r}$ such that

$$
K_{r}=T\left(\alpha_{r}\right) \circ X_{r}+\sum_{\mu} \phi_{r}^{\mu} Y_{\mu}^{r} .
$$

Then, if $g \in C^{\infty}\left(P_{r+1}\right)$,

$$
K_{r} \cdot g=X_{r} \cdot \alpha_{r}^{*}(g)+\sum_{\mu}\left(Y_{\mu}^{r} \cdot g\right) \phi_{r}^{\mu} .
$$

For $r \geqslant 1$, the $Y_{\mu}^{r}$ satisfy

$$
Y_{\mu}^{r} \cdot \phi_{r+1}^{\mu} \underset{p_{r}^{\prime \prime}}{\widetilde{T}} \delta_{\mu}^{v} \text {. }
$$

The first result is obtained in coordinates by observing that each component of the vector-valued function $K_{r}-T\left(\alpha_{r}\right) \circ X_{r}$ vanishes on $P_{r}^{(1)}$, therefore it is a $C^{\infty}\left(P_{r}\right)$ linear combination of the primary constraints. To obtain (13.7), just apply (13.6) and the preceding proposition.

On the other hand there is also a connection between the intermediate evolution operator $K_{r}$ and the fields $X_{r+1}$.

Proposition 11: $X_{r+1}$ is a primary dynamical field in $P_{r+1}(0 \leqslant r \leqslant k-1)$ if and only if there exist $m$ functions $\lambda_{r}^{\mu}$ in $P_{r}$ such that

$$
K_{r}=X_{r+1}{ }^{\circ} \alpha_{r}+\sum_{\mu} \lambda_{r}^{\mu} \Gamma_{\mu}^{r+1} \circ \alpha_{r} .
$$

Then, if $g \in C^{\infty}\left(P_{r+1}\right)$,

$$
K_{r} \cdot g=\alpha_{r}^{*}\left(X_{r+1} \cdot g\right)+\sum_{\mu} \alpha_{r}^{*}\left(\Gamma_{\mu}^{r+1} \cdot g\right) \lambda_{r}^{\mu} .
$$


The functions $\lambda_{r}$, are uniquely determined by $X_{r+1}$ and are not $\alpha$, projectable. More precisely,

$$
\Gamma_{\mu}^{r} \cdot \lambda_{r}^{v}=\delta_{\mu}^{v} \text {. }
$$

Suppose that $X_{r+1}$ is a primary dynamical field. For $r=k-1(13.8)$ is a consequence of proposition 8. Otherwise it is just a matter of computing

$$
\begin{aligned}
& T\left(\alpha_{r+1}\right)^{\circ}\left(K_{r}-X_{r+1} \circ \alpha_{r}\right) \\
& \quad=\left(K_{r+1}-T\left(\alpha_{r+1}\right) \circ X_{r+1}\right)^{\circ} \alpha_{r}=0,
\end{aligned}
$$

where (7.1) and (13.4) have been used. Therefore, since the $\Gamma_{\mu}^{r+1}$ are a frame for $\operatorname{Ker} T\left(\alpha_{r+1}\right)$, there exist some uniquely determined functions $\lambda_{r}^{\mu}$ such that

$$
K_{r}-X_{r+1}^{\circ} \alpha_{r}=\sum_{\mu} \lambda_{r}^{\mu} \Gamma_{\mu}^{r+1} \circ \alpha_{r} .
$$

The same argument proves the converse if $r \neq k-1$. Otherwise, if

$$
K_{k-1}=X_{\breve{H}^{\circ}}^{\circ} \alpha_{k-1}+\sum_{\mu} \tilde{\lambda}_{k-1}^{\mu} X_{\phi_{k}^{\mu}}^{\circ} \alpha_{k-1},
$$

then $X_{k}=X_{P_{k}^{\prime \prime 1}}$, with $H \underset{P_{k}^{(1)}}{=} \widetilde{H}$ appropriate.

Finally, to prove the last assertion one can compute

$$
\Gamma_{\mu}^{r} \cdot\left(K_{r} \cdot g\right)=\sum_{\mu} \alpha_{r}^{*}\left(\Gamma_{v}^{r+1} \cdot g\right)\left(\Gamma_{\mu}^{r} \cdot \lambda_{r}^{v}\right),
$$

which is also $\alpha_{r}^{*}\left(\Gamma_{\mu}^{r+1} \cdot g\right)$ by $(9.5)$; then take into account the arbitrariness of $g$.

\section{SOME PARTICULAR PRIMARY DYNAMICAL FIELDS}

In order to perform an explicit stabilization algorithm in $P_{r}(0 \leqslant r \leqslant k)$ a particular primary dynamical field $\widetilde{X}_{r}$ is needed to start, in the same way that a Hamiltonian function is needed to develop the Dirac-Bergman constraint algorithm.

Since we are only interested in the values of the primary dynamical fields in $P_{r}^{(1)}$, we can restrict ourselves to look for dynamical fields of the form (from now on the summation convention over the greek indices is assumed)

$$
X_{r}=\widetilde{X}_{r}+\eta_{r}^{\mu} \Gamma_{\mu}^{r} .
$$

They are known except for the arbitrariness of functions $\eta_{r}^{\mu}$. The requirement of tangency to the primary submanifold (which is needed in order to have integral curves on it) can determine some of these functions and can also lead to new constraints. This is the beginning of the stabilization algorithm that will be treated in detail in the next section.

Suppose that $H$ and $\phi_{k}^{\mu}$ have been chosen. For $r=k$ we simply put $\widetilde{X}_{k}=X_{H}$. Otherwise, using coordinates equation (11.5) determines all the components of $X_{r}$ except $\tilde{v}^{2 k-1-r}$, the coefficient of $\partial / \partial q^{2 k-1-r}$. The general solution of (11.5c) will be obtained in $P_{r}^{(1)}$ by adding to a particular one $\tilde{v}^{2 k-1-r}$ the vectors $\eta_{r}^{\mu} \gamma_{\mu}$ of $\operatorname{Ker} W$, where $\eta_{r}^{\mu}$ will be taken as arbitrary functions of time.

Let us look for a particular $\tilde{v}^{2 k-1-r}$. Take Eq. (12.4) and consider the coefficients of $\partial / \partial q^{k-1}$ :

$q^{k}=\alpha_{k-1}^{*}\left(\frac{\partial H}{\partial p_{k-1}}\right)+\lambda_{k-1}^{\mu}\left(q^{0}, \ldots, q^{k}\right) \alpha_{k-1}^{*}\left(\frac{\partial \phi_{k}^{\mu}}{\partial p_{k-1}}\right)$.
Derivation with respect $q^{\kappa}$ gives a "completeness relation" 43

$$
\delta_{c}^{a}=M^{a h} W_{b c}+\gamma_{\mu}^{a} \frac{\partial \lambda_{k-1}^{\mu}}{\partial q^{k, c}}
$$

where

$$
\begin{aligned}
M:= & \alpha_{k-1}^{*}\left(\frac{\partial^{2} H}{\partial p_{k-1} \partial p_{k-1}}\right) \\
& +\lambda_{k-1}^{\mu} \alpha_{k-1}^{*}\left(\frac{\partial^{2} \phi_{k}^{\mu}}{\partial p_{k-1} \partial p_{k-1}}\right) .
\end{aligned}
$$

(For sake of readability indices of coordinates in $Q$ appear explicitly in this equality.)

Then, use of (14.2) allows us to write $(11.5 \mathrm{c})$ in the form

$$
v^{2 k-1-r}=\tilde{v}^{2 k-1-r}+\gamma_{\mu}\left(\frac{\partial \lambda_{k-1}^{\mu}}{\partial q^{k}} v^{2 k-1-r}\right),
$$

where

$$
\begin{aligned}
\tilde{v}^{2 k-1-r}= & (-1)^{k-1-r} M \\
& \times\left(\frac{\partial L}{\partial q^{r}}-p_{r-1}-\sum_{i=0}^{2 k-2-r} \frac{\partial \hat{p}_{r}}{\partial q^{i}} q^{i+1}\right) .
\end{aligned}
$$

In this way a particular primary dynamical vector field $\widetilde{X}_{r}$, which is coordinate depending, has been defined. For this particular $\widetilde{X}_{r}$ the propositions in the preceding section hold, with particular values of $\lambda$ and $Y$.

Proposition 12: For $1 \leqslant r \leqslant k-1$, the equality

$$
K_{r-1}=\widetilde{X}_{r} \circ \alpha_{r-1}+\lambda_{r-1}^{\mu} \Gamma_{\mu}^{r} \circ \alpha_{r-1}
$$

holds, with the $m$ functions

$$
\lambda_{r-1}^{\mu}\left(q^{0}, \ldots, q^{k}, q^{2 k-r}\right)=\frac{\partial \lambda_{k-1}^{\mu}}{\partial q^{k}} q^{2 k-r},
$$

and $\lambda_{k-1}^{\mu}$ defined in Proposition 8 .

The only point to show is that

$$
q^{2 k-r}=\alpha_{r-1}^{*}\left(\tilde{v}^{2 k-1-r}\right)+\gamma_{\mu} \lambda_{r-1}^{\mu},
$$

which are the coefficients of the highest velocities in both hands, and this is obtained using (14.4), (3.5), (3.3), and (14.2).

$$
\begin{aligned}
& \text { Proposition 13: For } 0 \leqslant r \leqslant k-1 \text { the equality } \\
& K_{r}=T\left(\alpha_{r}\right) \circ \widetilde{X}_{r}+\phi_{r}^{\mu} Y_{\mu}^{r},
\end{aligned}
$$

holds, with the $m$ vector fields along $\alpha_{r}$

$$
\begin{aligned}
Y_{\mu}^{r} & =(-1)^{k-1-r} \frac{\partial \lambda_{k-1}^{\mu}}{\partial q^{k}} \frac{\partial}{\partial p_{r-1}} \\
& =(-1)^{k-1-r} \frac{\partial \lambda_{r}^{\mu}}{\partial q^{2 k-1-r}} \frac{\partial}{\partial p_{r-1}} .
\end{aligned}
$$

It suffices to compute the local expression of $K_{r}-T\left(\alpha_{r}\right) \circ \widetilde{X}_{r}$, using (3.3), (14.4), (14.2), and (13.3).

\section{STABILIZATION ALGORITHMS AND RELATIONS BETWEEN THEM}

In every space $P_{r}(0 \leqslant r \leqslant k)$ we have dynamical fields $X_{r}=\widetilde{X}_{r}+\eta_{r}^{\mu_{1}} \Gamma_{\mu_{1}}^{r}$, where $\eta_{r}^{\mu_{1}}$ are, in principle, $m_{1}=m$ arbitrary functions of time. (From now on the previously used greek indices acquire a subscript " 1 " which refers to the first 
level of the stabilization algorithm.) We are interested in integral curves for $X_{r}$ only on the submanifold of primary constraints $P_{r}^{(1)}$. This implies a new requircment, namely the tangency of $X_{r}$ to $P_{r}^{(1)}$, which leads, as it is well known in the first-order case, to the determination of some functions $\eta$ and also to new constraints, the secondary constraints. In this way a new submanifold $P_{r}^{(2)} \subset P_{r}^{(1)}$ is defined. The procedure continues by requiring again tangency of $X_{r}$ to the new submanifold and so on. This is the step-by-step stabilization algorithm for $P_{r}$. Sometimes all the constraints are so obtained but the primary ones will be loosely called secondary constraints.

Now we are going to show the intimate connections between the stabilization algorithms for all the spaces $P_{r}$ $(0 \leqslant r \leqslant k)$. As a particular case the connection between the Hamiltonian stabilization algorithm in $P_{k}$ and the Lagrangian one in $P_{\mathrm{o}}$ will be revealed.

Actually, the connection between the primary constraints provided by the operators $K_{r}$ will be reproduced at each step of the stabilization algorithm. Let us first introduce two results.

Proposition 14: If $\psi_{r} \in C^{\infty}\left(P_{r}\right)$ is a constraint, then $\alpha_{r-1}^{*}\left(\psi_{r}\right)$ is also.

Given a solution $\xi_{r-1}$ of the equations of motion in $P_{r-1}$, we know from Sec. $\mathrm{X}$ that $\xi_{r}:=\alpha_{r-1} 0^{\circ} \xi_{r-1}$ is a solution of the equations of motion in $P_{r}$, therefore $\left(\alpha_{r-1}^{*}\left(\psi_{r}\right)\right) \circ \xi_{r-1}=\psi_{r} \circ \xi_{r}=0$.

Proposition 15: If $\psi_{r} \in C^{\infty}\left(P_{r}\right)$ is a constraint, then $K_{r-1} \cdot \psi_{r}$ is also.

Use (10.3) with $g=\psi_{r}$.

Let us consider the stabilization algorithm for the Hamiltonian formalism. Its first step leads to a splitting ${ }^{43.58}$ of the primary constraints $\phi_{k}^{\mu_{1}}\left(1 \leqslant \mu_{1} \leqslant m_{1}\right)$ of $P_{k}$ into a set of primary first class constraints $\phi_{k}^{\mu_{1}^{\prime}}\left(1 \leqslant \mu_{1}^{\prime} \leqslant m_{1}^{\prime}\right)$ and a set of primary second class constraints $\phi_{k}^{\mu_{1}^{\prime \prime}}\left(m_{1}^{\prime}+1 \leqslant \mu_{1}^{\prime \prime} \leqslant m_{1}\right)$. They are characterized by the properties

$$
\begin{aligned}
& \Gamma_{\mu_{1}}^{k} \cdot \phi_{k}^{\mu_{p_{k}^{\prime}}^{\mu^{\prime \prime}}}=0, \\
& \Gamma_{\mu_{1}^{\prime}}^{k} \cdot \phi_{k}^{\mu_{i}^{\prime \prime}} \underset{P_{k}^{\prime \prime \prime}}{=} 0, \\
& \operatorname{det}\left(\Gamma_{\mu_{i}^{\prime \prime}}^{k} \cdot \phi_{k}^{\nu_{i}^{\prime \prime}}\right) \neq 0 .
\end{aligned}
$$

(In our local approach, this unequality is to hold in a neighborhood in $P_{k}$ of $P_{k}^{(1)}$.) The evolution vector fields then read:

$$
X_{k}=\widetilde{X}_{k}+\eta_{k}^{\mu_{i}^{i}} \Gamma_{\mu_{i}^{\prime}}^{k}+\eta_{k}^{\mu_{i}^{\prime \prime}} \Gamma_{\mu_{i}^{\prime \prime}}^{k}
$$

The tangency of $X_{r}$ to $P_{k}^{(1)}$ amounts to saying $X_{k} \cdot \phi_{k}^{\mu_{1}}=0$. Then, taking (15.1) into account, stability for $\phi_{k}^{\mu_{i}^{\prime \prime}}$ "determines" $\eta_{k}^{\mu_{i}^{\prime \prime}}=f_{k}^{\mu_{i}^{\prime \prime}} \in C^{\infty}\left(P_{k}\right)$ by requiring

$$
0=\widetilde{X}_{k} \cdot \phi_{k}^{\mu_{1}^{\prime \prime}}+f_{k}^{v_{1}^{\prime \prime}} \Gamma_{v_{1}^{\prime \prime}}^{k} \cdot \phi_{k}^{\mu_{1}^{\prime \prime}} \text {. }
$$

[In fact, Eq. (15.3) needs only hold in $P_{k}^{(1)}$, therefore the functions $f_{k}^{\nu_{1}^{\prime \prime}}$ are truly determined up to primary constraints. ] On the other hand, stability of constraints $\phi_{k}^{\mu_{i}^{\prime}}$ pro- duces the new constraints

$$
\phi_{k}^{\mu_{2}}:=\widetilde{X}_{k} \cdot \phi_{k}^{\mu_{i}^{i}},
$$

where $\mu_{2}$ runs over the same values as $\mu_{k}^{\prime}$ but labels secondary constraints.

It is easy to see how this procedure in $P_{k}$ applies to $P_{k-1}$. Consider, from Proposition 9,

$$
\begin{aligned}
& \phi_{k-1}^{\mu_{i}^{\prime}}=K_{k-1} \cdot \phi_{k}^{\mu_{i}^{i}}, \\
& \phi_{k-1}^{\mu_{3}^{\prime}}=K_{k-1} \cdot \phi_{k}^{\mu_{i}^{\prime \prime}} .
\end{aligned}
$$

The evolution vector field in $P_{k}, 1$ is

$$
X_{k-1}=\widetilde{X}_{k-1}+\eta_{k-1}^{\mu_{i}^{i}} \Gamma_{\mu_{1}^{\prime}}^{k-1}+\eta_{k-1}^{\mu_{i}^{*}} \Gamma_{\mu_{1}^{\prime r}}^{k-1}
$$

Stability for $\phi_{k-1}^{\mu_{i}^{\prime \prime}}$ determines $\eta_{k-1}^{\mu_{1}^{\prime \prime}}=f_{k-1}^{\mu_{i}^{*}} \in C^{\infty}\left(P_{k-1}\right)$ by requiring

$$
0=\widetilde{X}_{k-1} \cdot \phi_{k-1}^{\mu_{1}^{\prime \prime}}+f_{k-1}^{\nu_{1}^{\prime \prime}} \Gamma_{v_{1}^{\prime \prime}}^{k-1} \cdot \phi_{k-1}^{\mu_{1}^{\prime \prime}},
$$

where use has been made of (9.5) and (15.1c) to show that

$$
\begin{aligned}
\operatorname{det}\left(\Gamma_{v_{i}^{\prime \prime}}^{k-1} \cdot \phi_{k-1}^{\mu_{i}^{\prime \prime}}\right) & =\operatorname{det}\left(\Gamma_{v_{i}^{\prime \prime}}^{k-1} \cdot\left(K_{k-1} \cdot \phi_{k}^{\mu_{1}^{\prime \prime}}\right)\right) \\
& =\operatorname{det}\left(\alpha_{k-1}^{*}\left(\Gamma_{v_{i}^{\prime \prime}}^{k} \cdot \phi_{k}^{\mu_{1}^{\prime \prime}}\right)\right) \\
& =\alpha_{k-1}^{*}\left(\operatorname{det}\left(\Gamma_{v_{i}^{\prime \prime}}^{k} \cdot \phi_{k}^{\mu_{i}^{\prime \prime}}\right)\right) \\
& \neq 0 .
\end{aligned}
$$

Stability of the constraints $\phi_{k-1}^{\mu_{i}}$ produces the new constraints

$$
\phi_{k-1}^{\mu_{2}}:=\widetilde{X}_{k-1} \cdot \phi_{k-1}^{\mu_{1}^{\prime}},
$$

where $\mu_{2}$ runs over the same values as $\mu_{1}^{\prime}$ but labels secondary constraints, now in $P_{k-1}$. Then, using (13.6), the definition of the secondary Hamiltonian constraints, (13.9), (15.1a) and the definition of the primary constraints of $P_{k-1,}$

$$
\begin{aligned}
K_{k-1} \cdot \phi_{k}^{\mu_{2}} & =\widetilde{X}_{k-1}^{(1)} \cdot \alpha_{k-1}^{*}\left(\phi_{k}^{\mu_{2}}\right) \\
& =\widetilde{X}_{k-1} \cdot \alpha_{k-1}^{*}\left(\widetilde{X}_{k} \cdot\left(\phi_{k}^{\mu_{1}^{\prime}}\right)\right) \\
& =\widetilde{X}_{k-1} \cdot\left(K_{k-1} \cdot \phi_{k}^{\mu_{i}^{i}}\right) \\
& =\widetilde{X}_{k-1} \cdot \phi_{k-1}^{\mu_{i}^{i}} \\
& =\phi_{k-1}^{\mu_{2}} .
\end{aligned}
$$

The same reasoning applies to every intermediate space $P_{r}$. Relations (15.1) read, for $r$ instead of $k$,

$$
\begin{aligned}
& \Gamma_{\mu_{1}}^{r} \cdot \phi_{r}^{\mu_{i}^{i}}=0, \\
& \Gamma_{p_{r}^{\prime \prime \prime}}^{r} \cdot \phi_{r}^{\mu_{i}^{\prime \prime}} \underset{p_{r}^{\prime \prime \prime}}{=} 0, \\
& \operatorname{det}\left(\Gamma_{\mu_{i}^{\prime \prime}}^{r} \cdot \phi_{k}^{v_{i}^{\prime \prime}}\right) \neq 0,
\end{aligned}
$$

Some arbitrary functions are determined by $\eta_{r}^{\nu^{\prime \prime}}=f_{r}^{v_{i}^{*}}$ from

$$
0=\widetilde{X}_{r} \cdot \phi_{r}^{\mu_{1}^{\prime \prime}}+f_{r}^{\nu_{1}^{\prime \prime}} \Gamma_{v_{i}^{\prime \prime}}^{r} \cdot \phi_{r}^{\mu_{i}^{*}}
$$

The constraints 


$$
\phi_{r}^{\mu_{2}}:=\widetilde{X}_{r} \cdot \phi_{r}^{\mu_{i}^{\prime}}
$$

define $P_{r}^{(2)} \subset P_{r}^{(1)}$ and the following relation holds:

$$
\phi_{r-1}^{\mu_{2}} \underset{p !(1)}{=} K_{r-1} \cdot \phi_{r}^{\mu_{2}}
$$

The stabilization algorithm runs, therefore, in a parallel way in every space $P_{r}$.

The next step, which is to define $P_{r}^{(3)}$, goes again in the same fashion. During this procedure some of the previously first-class primary Hamiltonian constraints may become second class. At the same time the evolution vector fields $X_{r}$ have lost part of their arbitrariness thanks to the determination of the corresponding functions $\eta_{r}^{\mu_{1}^{\prime \prime}}=f_{r}^{\mu_{1}^{\prime \prime}}$. Notice that the number of arbitrary functions which are determined in any step of the stabilization algorithm is the same in each intermediate space.

Our conclusion is that the operator $K_{r-1}$ relates, step by step, the stabilization algorithms of $P_{r}$ and $P_{r-1}$, for every $1<r \leqslant k$. Therefore, we have the following.

Proposition 16: If the constraints $\phi_{r}^{\mu_{l}}$ define $P_{r}^{(l)} \subset P_{r}^{(l-1)}$ $(1 \leqslant r \leqslant k)$, then the constraints $K_{r} \cdot \phi_{r}^{\prime \prime}$ define $P_{r-1}^{(l)} \subset P_{r-1}^{(l-1)}$.

Direct consequence of this result is the following theorem.

Theorem 8: All the constraints of space $P_{r-1} \quad(1 \leqslant r \leqslant k)$ can be written in the form $K_{r-1} \cdot \phi_{r}$, where $\phi_{r}$ runs over the constraints of space $P_{r}$. In particular, all the Lagrangian constraints can be obtained by applying the composition of the $k$ differential operators $K_{k-1}, \ldots, K_{0}$ to all the Hamiltonian constraints.

This theorem is, in some sense, the "inverse" of Proposition 15. Instead, Proposition 14 has no "inverse" because there generally exist constraints which are not $\alpha_{r-1}$ projectable. The whole situation is described by the following result.

Proposition 17: If the constraints $\phi_{r}^{\mu_{l}}\left(1 \leqslant \mu_{l} \leqslant m_{l}\right)$ define the submanifold $P_{r}^{(1)} \subset P_{r}^{(l-1)}$, and if the functions $f_{r}^{\mu_{i-1}^{*}}$ $\left(1<\mu_{-1}^{\prime \prime} \leqslant m_{l-1}^{\prime \prime}\right)$ are the functions determined in the $l$ th step of the algorithm (to obtain $P_{r}^{(l)}$ from $P_{r}^{(l-1)}$ ), then $P_{r-1}^{(l-1)}$ is defined in $P_{r-1}^{(l-2)}$ by

$$
\begin{aligned}
& \alpha_{r-1}^{*}\left(\phi_{r}^{\mu_{l}}\right), \quad 1 \leqslant \mu_{l} \leqslant m_{l}, \\
& \chi_{r-1}^{\mu_{i-1}^{\prime \prime}:}=\lambda_{r-1}^{\mu_{l-1}^{\prime \prime}}-\alpha_{r-1}^{*}\left(f_{r}^{\left.\mu_{-1}^{\prime \prime}\right), \quad 1 \leqslant \mu_{l-1}^{\prime \prime} \leqslant m_{l-1}^{\prime \prime} .}\right.
\end{aligned}
$$

This result will be demonstrated for the level $l=2$, the general case can be analogously obtained by a similar reasoning and use of induction on $l$.

We recall that $P_{r}^{(2)} \subset P_{r}^{(1)}$ is defined by (15.9). Now consider $P_{r-1}^{(1)}$. According to (15.10), this submanifold is defined by the constraints $K_{r-1} \cdot \phi_{r}^{\mu_{1}}$. The splitting $\left(\mu_{1}\right)=\left(\mu_{1}^{\prime}, \mu_{1}^{\prime \prime}\right)$, characterized by properties (15.7), allows to compute, using (13.9),

$$
K_{r-1} \cdot \phi_{r}^{\mu_{1}^{\prime}}=\alpha_{r-1}^{*}\left(\widetilde{X}_{r} \cdot \phi_{r}^{\mu_{1}^{\prime}}\right)+\lambda_{r-1}^{v_{1}} \alpha_{r-1}^{*}\left(\Gamma_{v_{1}}^{r} \cdot \phi_{r}^{\mu_{1}^{\prime}}\right) ;
$$

but $\Gamma_{v_{1}}^{r} \cdot \phi_{r}^{\mu_{1}^{\prime}} \underset{P_{r}^{(1)}}{=} 0$ implies $\alpha_{r-1}^{*}\left(\Gamma_{v_{1}}^{r} \cdot \phi_{r}^{\mu_{1}^{\prime}}\right)=0$, therefore

$$
K_{r-1} \cdot \phi_{r}^{\mu_{1}^{\prime}}=\alpha_{r-1}^{*}\left(\widetilde{X}_{r} \cdot \phi_{r}^{\mu_{1}^{\prime}}\right)=\alpha_{r-1}^{*}\left(\phi_{r}^{\mu_{2}}\right)
$$

(index $\mu_{2}$ substitutes $\mu_{1}^{\prime}$ for secondary constraints).

On the other hand, since $\alpha_{r-1}^{*}\left(\Gamma_{\nu_{1}^{\prime}}^{r} \cdot \phi_{r}^{\mu_{1}^{\prime \prime}}\right)=0$,

$$
\begin{aligned}
K_{r-1} \cdot \phi_{r}^{\nu_{r}^{\prime \prime}} & =\alpha_{r-1}^{*}\left(\tilde{X}_{r} \cdot \phi_{r}^{\nu_{r}^{\prime \prime}}\right)+\lambda_{r-1}^{\mu_{1}} \alpha_{r-1}^{*}\left(\Gamma_{\mu_{1}}^{r} \cdot \phi_{r}^{\nu_{r}^{\prime \prime}}\right) \\
& =\alpha_{r-1}^{*}\left(\tilde{X}_{r} \cdot \phi_{r}^{\nu_{1}^{\prime \prime}}\right)+\lambda_{r-1}^{\mu_{1}^{\prime \prime}} \alpha_{r-1}^{*}\left(\Gamma_{\mu_{1}^{\prime \prime}}^{r} \cdot \phi_{r}^{\nu_{1}^{\prime \prime}}\right)
\end{aligned}
$$

Then, use of (15.8) gives

$$
K_{r-1} \cdot \phi_{r}^{\nu_{1}^{\prime \prime}}=\left(\lambda_{r-1}^{\nu_{1}^{\prime \prime}}-\alpha_{r-1}^{*}\left(f_{r}^{v_{1}^{\prime \prime}}\right)\right) \alpha_{r-1}^{*}\left(\Gamma_{v_{1}^{\prime \prime}}^{r} \cdot \phi_{r}^{\mu_{1}^{\prime \prime}}\right),
$$

and considering (15.7) we conclude that $K_{r-1} \cdot \phi_{r}^{v_{1}^{\prime \prime}}=0$ $\left(m_{1}^{\prime}+1 \leqslant \nu_{1}^{\prime \prime} \leqslant m_{1}\right)$ is equivalent to $\lambda_{r-1}^{\nu_{1}^{\prime \prime}}-\alpha_{r-1}^{*}\left(f_{r}^{\nu_{1}^{\prime \prime}}\right)$ $=0\left(m_{1}^{\prime}+1 \leqslant v_{1}^{\prime \prime} \leqslant m_{1}\right)$.

Use of (15.13) and (15.14) shows, therefore, that $P_{r}^{(1)}$ is defined by

$$
\begin{aligned}
& \alpha_{r-1}^{*}\left(\phi_{r}^{\mu_{2}}\right)=0, \quad 1 \leqslant \mu_{2} \leqslant m_{2}, \\
& \chi_{r-1}^{\mu_{1}^{\prime \prime}}:=\lambda_{r-1}^{\mu_{1}^{\prime \prime}}-\alpha_{r-1}^{*}\left(f_{r}^{\mu_{1}^{\prime \prime}}\right)=0, \quad m_{1}^{\prime}+1 \leqslant \mu_{1}^{\prime \prime} \leqslant m_{1},
\end{aligned}
$$

which finishes the proof.

This can be summarized by the following theorem.

Theorem 9: All the constraints of space $P_{r-1}(1 \leqslant r \leqslant k)$ can be written in the form $\alpha_{r-1}^{*}\left(\phi_{r}\right)$, where $\phi_{r}$ runs over the secondary constraints of space $P_{r}$, or in the form $\lambda_{r-1}-\alpha_{r-1}^{*}\left(f_{r}\right)$, where $f_{r}$ runs over the determined functions of the dynamics in $P_{r}$ and the $\lambda_{r-1}$ are the corresponding functions in $P_{r-1}$.

Notice that every determination of an arbitrary function in $P_{r}$ gives rise to a constraint of the form (15.12) in $P_{r-1}$. These constraints are not $\alpha_{r-1}$ projectable because $\Gamma \cdot \chi=\delta$, and the theorem therefore classifies the constraints of every $P_{r}(0 \leqslant r \leqslant k-1)$ into two sets, according to their $\alpha_{r}$ projectability.

Observe also that the stabilization of the $\alpha_{r}$-projectable constraints will lead (in general) to new constraints, which constitute the next level of constraints, whereas the stabilization of the non- $\alpha_{r}$-projectable constraints will lead to the determination of so many of the (previously) arbitrary functions as is the number of these constraints. All these results were already known for a first-order Lagrangian. ${ }^{36}$

At this point, the structure of constraints in spaces $P_{r}$ has been completely revealed: 


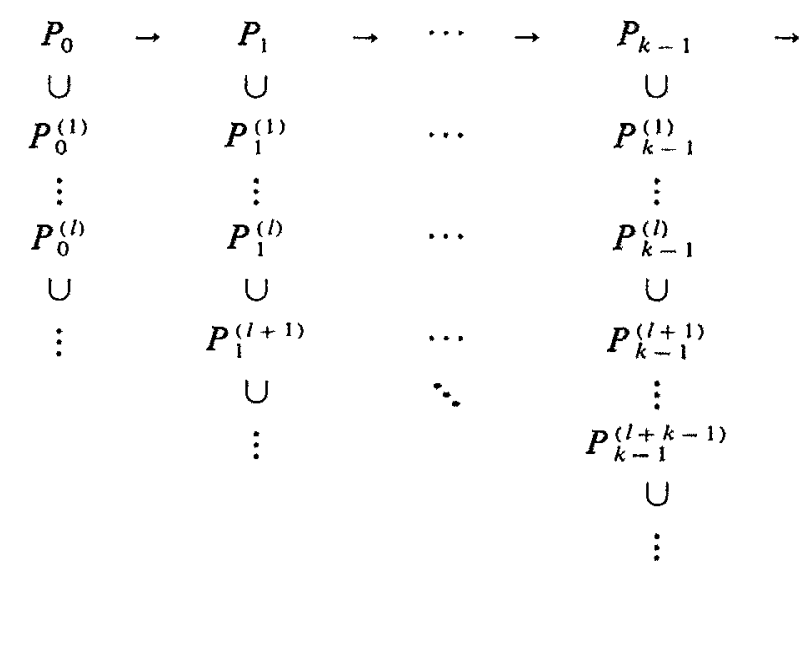

and the following corollary holds.

Proposition 18: For $0 \leqslant r \leqslant k-1$ and $l \geqslant 0$

$\alpha_{r}\left(P_{r}^{(l)}\right)=P_{r+1}^{(l+1)}$.

$\left(P_{r}^{(0)}\right.$ stands for $\left.P_{r}\right)$. In particular $P_{k}^{(k)}$ is identified as the image in $P_{k}$ of the Legendre-Ostrogradskir's transformation.

The proof goes by induction on $l$. For $l=0$ this is just the definition of $P_{r}^{(1)}$. Now suppose we know that $\alpha_{r}\left(P_{r}^{(l)}\right)=P_{r+1}^{(l+1)}$, from which $\alpha_{r}\left(P_{r}^{(l+1)}\right)=P_{r+1}^{(l+2)}$ must be deduced.

The submanifold $P_{r+1}^{(l+2)} \subset P_{r+1}^{(l+1)}$ is described by the constraints $\phi_{r+1}^{\mu_{l+2}}\left(1 \leqslant \mu_{l+2} \leqslant m_{l+2}\right)$. And from the preceding theorem the submanifold $P_{r}^{(l+1)} \subset P_{r}^{(l)}$ is described by the constraints $\alpha_{r}^{*}\left(\phi_{r+1}^{\mu_{t+2}}\right) \quad\left(1 \leqslant \mu_{l+2} \leqslant m_{t+2}\right)$ and $\chi_{r}^{\mu_{i+1}^{*}}$ $\left(m_{l+1}^{\prime}+1 \leqslant \mu_{l+1}^{\prime \prime} \leqslant m_{l+1}\right)$. But these last constraints are not $\alpha_{\mathrm{r}}$ projectable, so they do not contribute to restrict the image of $P_{r}^{(l+1)}$ under $\alpha_{r}$. Therefore, $\alpha_{r}\left(P_{r}^{(l+1)}\right)$ $=\alpha_{r}\left(P_{r}^{(l+1)^{\prime}}\right)$, where $P_{r}^{(l+1)^{\prime}}$ is defined in $P_{r}^{(l)}$ by the $\alpha_{r^{-}}$ projectable constraints $\alpha_{r}^{*}\left(\phi_{r+1}^{\mu_{l+2}}\right)\left(1 \leqslant \mu_{l+2} \leqslant m_{l+2}\right)$. Then, by its own definition, $\alpha_{r}\left(P_{r}^{(l+1)}\right)=P_{r+1}^{(l+2)}$.

\section{THE DETERMINATION OF "ARBITRARY" FUNCTIONS}

In the preceding section the close relation between the stabilization algorithms of the various intermediate spaces has been displayed. The established parallelism can actually be extended to the determination of some of the arbitrary functions of the corresponding dynamics. In fact, the operators $K_{r}$ give a relation between the determined functions $f_{r}^{\mu_{r}^{\prime \prime}}$ of the different intermediate spaces $P_{r}$. Consider the constraints in $P_{r}$

$$
\chi_{r}^{\mu_{r}}=\lambda_{r}^{\mu_{t}}-\alpha_{r}^{*}\left(f_{r+1}^{\mu_{r}}\right) .
$$

Then, by Proposition 15, $K_{r-1} \cdot \chi_{r}^{\mu_{r}^{\prime r}}$ will give constraints in $P_{r-1}$. They are, using (13.9),

$$
\begin{aligned}
K_{r-1} \cdot \chi_{r}^{\mu_{t}^{\prime \prime}} & =K_{r-1} \cdot \lambda_{r}^{\mu_{i}^{\prime \prime}}-K_{r-1} \cdot \alpha_{r}^{*}\left(f_{r+1}^{\mu_{i}^{\prime \prime}}\right) \\
& =\lambda_{r-1}^{\mu_{r}^{\prime \prime}}+\alpha_{r-1}^{*}\left(\widetilde{X}_{r} \cdot \lambda_{r}^{\mu_{r}^{\prime \prime}}\right)-\alpha_{r-1}^{*}\left(K_{r} \cdot f_{r+1}^{\mu_{r}^{\prime \prime}}\right) .
\end{aligned}
$$

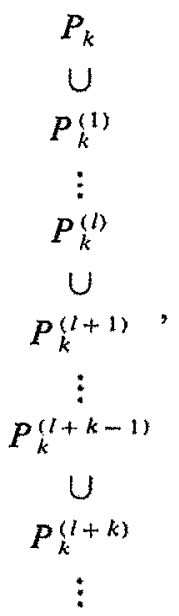

Therefore, up to constraints, we determine, for $1 \leqslant r \leqslant k-1$,

$$
\begin{aligned}
f_{r}^{\mu_{r}^{\prime \prime}} & =K_{p_{r}^{(r)}} K_{r} \cdot f_{r+1}^{\mu_{l}^{\prime \prime}}-\widetilde{X}_{r} \cdot \lambda_{r}^{\mu_{r}^{\prime \prime}} \\
& \underset{p_{r}^{\prime r}}{=} K_{r} \cdot f_{r+1}^{\mu_{i}^{\prime \prime}}-\sum_{i=0}^{2 k-2-r} q^{i+1} \frac{\partial \lambda_{r}^{\mu_{i}^{\prime \prime}}}{\partial q^{i}},
\end{aligned}
$$

where the last step is justified (Ref. 45, the Appendix) because $\tilde{v}^{2 k-1-r} \partial \lambda_{r}^{\mu_{1}} / \partial q^{2 k-1-r} \underset{p_{r}^{(1)}}{=} 0$.

\section{GAUGE FIXING AND DEGREES OF FREEDOM}

The stabilization algorithm in each space $P_{r}(0 \leqslant r \leqslant k)$ leads to a final constraint submanifold $P_{r}^{(F)}$. Constraints defining $P_{r}^{(f)}$ have been classified in Sec. VII into $\alpha_{r}$-projectable and non $-\alpha_{r}$-projectable constraints. Owing to this fact, it is easy to relate the dimensions of the different final submanifolds $P_{r}^{(F)}$.

Let $n_{r}$ be the number of independent constraints defining $P_{r}^{(F)} \subset P_{r}$. We recall that the number of primary constraints in each $P_{r}$ is $m_{1}$, except for $r=0$. Now consider $P_{r-1}$. Its number of independent $\alpha_{r-1}$ projectable constraints is $n_{r}-m_{1}$ because the pull-back of primary constraints of $P_{r}$ gives no relations in $P_{r-1}$, whereas the pullback of secondary constraints yields independent constraints in $P_{r-1}$.

The number of non- $\alpha_{r}$-projectable constraints in $P_{r-1}$ is just the number of functions $\eta_{r}^{t}$ in $X$, which are finally determined, $f_{r}^{\mu}$. Since every determination corresponds to a final second-class primary Hamiltonian constraint, this number is $m_{1}-m_{1}^{0}$, where $m_{1}^{0}$ is the number of the final first-class primary Hamiltonian constraints.

Thus we arrive, for $1 \leqslant r \leqslant k$, to $n_{r-1}=\left(n_{r}-m_{1}\right)+\left(m_{1}-m_{1}^{0}\right)=n_{r}-m_{1}^{0}$, and therefore

$$
n_{k}-n_{r}=(k-r) m_{1}^{0}, \quad 0 \leqslant r \leqslant k-1 .
$$

This proves the following.

Theorem 10: If $P_{r}^{(F)}$ is the final constraint submanifold of $P_{r}(0 \leqslant r \leqslant k)$, and if $m_{1}^{0}$ is the number of final first-class primary constraints in $P_{k}$, then the dimensions of these submanifolds are related by

$$
\operatorname{dim}\left(P_{r}^{(F)}\right)=\operatorname{dim}\left(P_{k}^{(F)}\right)+(k-r) m_{1}^{o} .
$$


In particular, $\operatorname{dim}\left(P_{0}^{(F)}\right)=\operatorname{dim}\left(P_{k}^{(F)}\right)+k m_{1}^{0}$.

Thus the final submanifolds of constraints have different dimensions depending on $P_{r}$. At first glance it could seem contradictory with our results of Secs. II and IV showing the equivalence between the dynamical contents of every space $P_{r}$. The way out of this puzzle is to consider that, due to gauge freedom, we have not yet determined the true number of degrees of freedom in every space $P_{r}$. Now we are going to introduce a gauge fixing procedure to determine this number, as it can be done for first-order Lagrangians. ${ }^{24}$

We start by introducing the gauge fixing constraints in $P_{k}$. The procedure is "propagated" afterwards to all the intermediate spaces.

Let us call point-gauge transformations those transformations that relate--in the space of a certain singular dynamical system - physically equivalent points, that is to say, points belonging to dynamical trajectories connected by gauge transformations. In the Hamiltonian formalism these transformations are generated by the vector fields associated (through the symplectic structure of phase space) to all the first-class constraints. ${ }^{22,24}$ We assume they are in number $n^{0}$. In order to make these transformations disappear, a gauge fixing constraint for each first-class constraint has to be introduced; this will rend all the constraints second class. Moreover, consistency requires the final Hamiltonian vector field to be tangent to the new submanifold of constraints. Owing to this reason, we shall proceed in two steps.

(1) Introduce $m_{1}^{0}$ primary gauge fixing constraints $F_{k}^{\mu_{1}^{0}}$ to convert all the first-class primary constraints into second class. So up to now remaining arbitrary functions $\eta_{k}^{\mu_{1}^{0}}$ of the dynamics are determined: $\eta_{k}^{\mu_{1}^{0}}=f_{k}^{\mu_{1}^{0}}$. Now dynamics is entirely fixed:

$$
X_{k}^{G F}=\widetilde{X}_{k}+f_{k}^{\mu_{1}} \Gamma_{\mu_{1}}^{k}
$$

( this corresponds to a Hamiltonian $H^{G F}=H+\Sigma_{\mu_{1}} f_{k}^{\mu_{1}} \phi_{k}^{\mu_{1}}$ ).

(2) Introduce the remaining $n^{0}-m_{1}^{0}$ secondary gauge fixing constraints $G_{k}^{\mu}$ under two requirements: (a) to convert all first-class secondary constraints into second class, and (b) to preserve the Hamiltonian as a first-class function:

$$
X_{k} \cdot G_{P_{k}^{(G)}}^{\mu}=0 .
$$

Once this gauge fixing procedure is performed, we arrive at a submanifold $P_{k}^{(G F)}$. This is the "initial conditions" submanifold. The total number of independent constraints becomes

$$
n_{k}^{G F}:=n_{k}+n^{0} \text {. }
$$

Now consider the preceding space $P_{k-1}$. Proposition 15 ensures that $F_{k-1}^{\mu_{1}^{0}}:=K_{k-1} \cdot F_{k}^{\mu_{1}^{0}}$ are gauge fixing constraints in $P_{k-1}$. These constraints realize the fixation of dynamics in $P_{k-1}$ in a parallel way as $F_{k}^{\mu_{9}^{9}}$ did in $P_{k}$. In fact, the final evolution vector field in $P_{k-1}^{(F)}$ is

$$
\begin{aligned}
X_{k-1}^{G F} & =\widetilde{X}_{k-1}+f_{k-1}^{\mu_{1}^{00}} \Gamma_{\mu_{1}^{00}}^{k-1}+\eta_{k-1}^{\mu_{1}^{0}} \Gamma_{\mu_{1}^{0}}^{k-1} \\
& =\widetilde{X}_{k-1}^{\prime}+\eta_{k-1}^{\mu_{1}^{0}} \Gamma_{\mu_{1}^{0}}^{k-1}
\end{aligned}
$$

and tangency of $X_{k-1}$ to the new submanifold of constraints determines $\eta_{k-1}^{\mu_{1}^{0}}=f_{k-1}^{\mu_{1}^{0}}$ by requiring

$$
0=\tilde{X}_{k-1} \cdot F_{k-1}^{\nu_{1}^{0}}+f_{k-1}^{\mu_{1}^{0}} \Gamma_{\mu_{1}^{0}}^{k-1} \cdot F_{k-1}^{\nu_{1}^{0}} \text {, }
$$

because, using (9.5),

$$
\begin{aligned}
\operatorname{det}\left(\Gamma_{\mu_{1}^{0}}^{k-1} \cdot F_{k-1}^{v_{1}^{0}}\right) & =\operatorname{det}\left(\Gamma_{\mu_{1}^{0}}^{k-1} \cdot\left(K_{k-1} \cdot F_{k}^{v_{1}^{0}}\right)\right) \\
& =\operatorname{det}\left(\alpha_{k-1}^{*}\left(\Gamma_{\mu_{1}^{0}}^{k} \cdot F_{k}^{v_{1}^{0}}\right)\right) \\
& =\alpha_{k-1}^{*}\left(\operatorname{det}\left(\Gamma_{\mu_{1}^{0}}^{k} \cdot F_{k}^{\nu_{1}^{0}}\right)\right) \\
& \neq 0 .
\end{aligned}
$$

Observe, however, that the constraints $F_{k}^{\mu_{1}^{0}}$ generate another type of constraints in $P_{k-1}$. Proposition 14 ensures that the functions $\alpha_{k-1}^{*}\left(F_{k}^{\mu_{1}^{0}}\right)$ are also constraints in $P_{k-1}$. It is easy to see that stability of these last constraints leads to constraints $F_{k-1}^{\mu_{1}^{0}}$ :

$$
\begin{aligned}
X_{k-1}^{G F} \cdot \alpha_{k-1}^{*}\left(F_{k}^{\mu_{1}^{0}}\right) & =\widetilde{X}_{k-1} \cdot \alpha_{k-1}^{*}\left(F_{k}^{\mu_{1}^{0}}\right) \\
& =K_{k-1} \cdot F_{k}^{\mu_{1}^{0}} \\
& =F_{k-1}^{\mu_{1}^{0}} C^{\prime}
\end{aligned}
$$

So we see that the first step of our gauge fixing procedure introduces $2 m_{1}^{0}$ constraints in $P_{k-1}$.

Now we study the consequences of the second step. Consider the $n^{0}-m_{1}^{0}$ gauge fixing constraints $\alpha_{k-1}^{*}\left(G_{k}^{\mu}\right)$ in $P_{k-1}$. Using (13.6) and (13.9), it is shown that these constraints are already stable:

$$
\begin{aligned}
X_{k-1}^{G F} \cdot \alpha_{k-1}^{*}\left(G_{k}^{v}\right) \underset{P_{k-1}^{(1)}}{=} & K_{k-1} \cdot G_{k}^{v} \\
= & \alpha_{k-1}^{*}\left(\widetilde{X}_{k-1} \cdot G_{k}^{v}\right) \\
& +\lambda_{k-1}^{\mu_{1}} \alpha_{k-1}^{*}\left(\Gamma_{\mu_{1}}^{k} \cdot G_{k}^{v}\right) \\
= & \alpha_{k-1}^{*}\left(X_{k-1}^{G F} \cdot G_{k}^{v}\right)+\left(\lambda_{k-1}^{\mu_{1}}\right. \\
& \left.-\alpha_{k-1}^{*}\left(f_{k}^{\mu_{1}}\right)\right) \alpha_{k-1}^{*}\left(\Gamma_{\mu_{1}}^{k} \cdot G_{k}^{v}\right)
\end{aligned}
$$

$$
\underset{P(O H)}{=} 0,
$$

where $P_{k-1}^{(G F)}$ is the submanifold defined by all the constraints, stabilization, and gauge fixing, in $P_{k-1}$.

Summing up, we have introduced $2 m_{1}^{0}+\left(n^{0}-m_{1}^{0}\right)$ gauge fixing constraints to define $P_{k-1}^{(G F)} \subset P_{k-1}^{(F)}$.

In $P_{k-2}$ the situation is quite analogous. Fixation of dynamics is achieved by requiring tangency of $X_{k-2}$ to the new submanifold of constraints defined by $F_{k-2}^{\mu_{1}^{0}}:=K_{k-2} \cdot F_{k-1}^{\mu_{1}^{0}}$. But two additional generations of constraints appear:

$$
\alpha_{k-2}^{*}\left(\alpha_{k-1}^{*}\left(F_{k}^{\mu_{1}^{0}}\right)\right)
$$

and

$$
\begin{aligned}
\alpha_{k-2}^{*}\left(F_{k-1}^{\mu_{1}^{0}}\right) & =\alpha_{k-2}^{*}\left(K_{k-1} \cdot F_{k}^{\mu_{1}^{0}}\right) \\
& =K_{k-2} \cdot \alpha_{k-1}^{*}\left(F_{k}^{\mu_{1}^{0}}\right) .
\end{aligned}
$$

Therefore, the first step of the gauge fixing procedure leads 
to $3 m_{1}^{0}$ constraints in $P_{k-2}$. The second step leads to $n^{0}-m_{1}^{0}$, just the pull-back through $\alpha_{k-2}$ of $\alpha_{k-1}^{*}\left(G_{k-1}^{v}\right)$. We conclude that the number of gauge fixing constraints in $P_{k-2}$ is $3 m_{1}^{0}+\left(n^{0}-m_{1}^{0}\right)$.

The procedure applies to all the spaces $P_{r}(0 \leqslant r \leqslant k)$. When we go from $P_{r}$ to $P_{r-1}$, the number of gauge fixing constraints increases in $m_{1}^{0}$. Then the final result is that the number of gauge fixing constraints in $P_{r}$ is $(k-r-1) m_{1}^{0}+\left(n^{0}-m_{1}^{0}\right)=(k-r) m_{1}^{0}+n^{0}$. Then, the total number of constraints (standard and gauge fixing) in $P_{r}$ is

$$
\begin{aligned}
n_{r}^{G F} & =n_{r}+(k-r) m_{1}^{0}+n^{0} \\
& =\left(n_{k}-(k-r) m_{1}^{0}\right)+(k-r)+n^{0} \\
& =n_{k}+n^{0} .
\end{aligned}
$$

Therefore, if the gauge fixing constraints define the submanifold $P_{r}^{(G F)} \subset P_{r}^{(F)}$, the following theorem holds.

Theorem 11: All the submanifolds $P_{r}^{(G F)}(0 \leqslant r \leqslant k)$ have the same dimension $2 k n-\left(n^{k}+n^{0}\right)$, where $n^{k}$ is the number of Hamiltonian constraints and $n^{0}$ the number of the final first-class ones. In particular, the higher-order Lagrangian and Hamiltonian formalisms have the same number of degrees of freedom.

\section{EXAMPLES}

We present here two simple examples to illustrate our results. Both are second-order Lagrangians.

\section{A. $L\left(x^{0}, x^{1}, x^{2}\right)=x^{2}$}

Here $Q=\mathbf{R}$, so the intermediate spaces are diffeomorphic to $\mathbf{R}^{4}$.

The momenta are trivially computed:

$$
\hat{p}_{1}=1, \quad \hat{p}_{0}=0 \text {. }
$$

The first one gives just one primary Hamiltonian constraint,

$$
\phi_{2}^{1}=1-p_{1} \text {, }
$$

which defines globally $\alpha_{1}\left(P_{1}\right) \subset P_{2}$ as a submanifold.

The energy in $P_{1}$ is $E_{1}=p_{0} x^{1}$ (so $E_{0}=0$ ). Therefore, a Hamiltonian can be globally defined

$$
H=p_{0} x^{1},
$$

up to primary Hamiltonian constraints. With this $H$ we compute the secondary Hamiltonian constraint

$$
\phi_{2}^{2}=\left\{\phi_{2}^{1}, H\right\}=p_{0} .
$$

This is first class with $\phi_{2}^{\prime}$ and $H$, therefore the Hamiltonian stabilization algorithm is finished and $P_{2}^{(F)}$ is diffeomorphic to $\mathbf{R}^{2}$.

Since the Hamiltonian constraints are first class, the constraints in $P_{1}$ are $\alpha_{1}$ projectable. Indeed there is just one constraint:

$$
\phi_{1}^{1}:=K_{1} \cdot \phi_{2}^{1}=p_{0}=\alpha_{1}^{*}\left(\phi_{2}^{2}\right) .
$$

Similarly, there are no Lagrangian constraints, so $P_{0}=P_{0}^{(F)}$.

Now let us consider the gauge freedom. $P_{2}^{(F)}$ is two dimensional and we have obtained two first-class Hamiltonian constraints. Therefore, the gauge fixing procedure leads to a zero-dimensional space: a point. We can take two gauge fixing constraints

$$
F_{2}=x^{1}, \quad G_{2}=x^{0} .
$$

The first one makes the primary constraint second class, and determines completely the dynamics: $H=p_{0} x^{1}$, up to quadratic primary constraints. $P_{1}$ :

Our procedure leads to three gauge fixing constraints in

$$
K_{1} \cdot F_{2}=x^{2}, \quad \alpha_{1}^{*}\left(F_{2}\right)=x^{1}, \quad \alpha_{1}^{*}\left(G_{2}\right)=x^{0} .
$$

In the same way, the gauge fixing constraints in $P_{0}$ are

$$
x^{3}, x^{2}, x^{1}, x^{0} .
$$

\section{3. $L\left(x^{0}, x^{1}, x^{2}, y^{0}, y^{4}, y^{2}\right)=x^{1} y^{2}$}

Here $Q=\mathbf{R}^{2}$, and the intermediate spaces are diffeomorphic to $\mathbf{R}^{8}$.

The momenta are

$$
\begin{aligned}
& \hat{p}_{x 1}=0, \quad \hat{p}_{x 0}=y^{2}, \\
& \hat{p}_{y 1}=x^{1}, \quad \hat{p}_{y 0}=-x^{2} .
\end{aligned}
$$

The submanifold $\alpha_{1}\left(P_{1}\right) \subset P_{2}$ is globally defined by the two primary Hamiltonian constraints,

$$
\phi_{2}=x^{1}-p_{y 1}, \quad \psi_{2}=p_{x 1} .
$$

On the other hand the energy in $p_{1}$ is $E_{1}=p_{x 0} x^{1}+p_{y 0} y^{1}$ (now $E_{0}=x^{1} y^{2}-y^{1} x^{2}$ ). Any Hamiltonian has the form $H=p_{x 0} x^{l}+p_{80} y^{1}+\lambda \phi_{2}+\mu \psi_{2}$. But the primary Hamiltonians are second class, so making $H$ first class determines its arbitrary functions, and we have to use

$$
H=p_{y 0}\left(y^{\prime}-p_{x 1}\right)+p_{x 0} p_{y 1},
$$

up to quadratic primary constraints. No more constraints are to be found, and no gauge fixing is required: $P_{2}^{(G F)}=P_{2}^{(F)}$, which is six dimensional.

Since all the Hamiltonian constraints arc second class, there are no projectable constraints in $P_{0}$ and $P_{1}, P_{1}^{(F)}$ is defined by

$$
\phi_{1}:=K_{1} \cdot \phi_{2}=x^{2}+p_{y 0}, \quad \psi_{1}:=K_{1} \cdot \psi_{2}=y^{2}-p_{y 0} .
$$

And the Lagrangian constraints, which define $P_{0}^{(F)}$, are

$$
\phi_{0}:=K_{0} \cdot \phi_{1}=x^{3}, \quad \psi_{0}:=K_{0} \cdot \psi_{1}=y^{3} .
$$

The final constraint submanifolds are therefore diffeomorphic to $\mathbf{R}^{6}$ in each formalism.

\section{CONCLUSIONS}

In this paper we have performed a detailed study of higher-order Lagrangians. As a first step, some geometric structures particular to higher-order formalism have been introduced: in particular, the definition of intermediate spaces allowing the decomposition of the Ostrogradskir's transformation into partial transformations. With them, several constructions already known for first-order Lagrangians have been extended to higher-order theories.

Next, all these spaces have been endowed with corresponding dynamics, which have been proven to be equivalent. This gives in particular the equivalence between the Lagrangian and the Hamiltonian formalisms. 
In the singular case, the structure of the constraints arising from the dynamics of every intermediate space is completely displayed and the connections between the different stabilization algorithms are shown. These connections can be performed in two different and complementary ways. As a consequence, the Lagrangian constraints can be easily and explicitly constructed from the Hamiltonian constraints.

It is also possible to relate the dimensions of all the constraint submanifolds. These dimensions are different when some primary Hamiltonian constraints are first class. In this case there is some gauge freedom: the various dynamics are not completely determined due to some arbitrary functions, and a gauge fixing procedure is needed to get rid of the superfluous degrees of freedom. A gauge fixing in the Hamiltonian formalism provides gauge fixings for the intermediate formalisms, in particular for the Lagrangian formalism. Then it is found that the true number of degrees of freedom (the dimension of the gauge fixed constraint submanifold) is the same either in the Lagrangian or in the Hamiltonian formalisms - or in any intermediate formalism.

It should be emphasized that, although we have not refused to use coordinates, we have presented most of our results in a geometric language.

'D. Hilbert, Nachr. Ges. Wiss. Göttingen (1915).

${ }^{2}$ H. Weyl, Time, Space, Matter (Dover, New York, 1952).

${ }^{3}$ B. A. Dubrovin, S. P. Novikov, and A. T. Fomenko, Géométrie contemporaine. Méthodes et Applications (Mir, Moscow, 1982).

${ }^{4} M$. Dresden and S. K. Dutt, "Pure gravity as a constrained second-order system," New York Univ. preprint ITP-SB-86-32.

${ }^{5}$ A. Polyakov, Nucl. Phys. B 268, 406 (1986).

' V. V. Nesterenko and N. Suan Han, Int. J. Mod. Phys. A 3, 2315 (1988).

${ }^{7}$ F. Bopp, Ann. Phys. 38, 345 (1940).

'B. Podolski and P. Schwed, Rev. Mod. Phys. 20, 40 (1948).

${ }^{9}$ C. A. P. Galvão and B. M. Pimentel, Can. J. Phys. 66, 460 (1988),

${ }^{10}$ C. A. P. Galvão, "A generalized Yang-Mills theory I: general aspects of the classical theory," preprint ISSN 0029-3865.

"K. S. Stelle, Phys. Rev. D. 16, 953 (1977)

${ }^{12}$ D. G. Boulware and S. Deser, Phys. Rev. Lett. 55, 2656 (1984).

"1.R. D. Pisarski, Phys. Rev. D 34, 670 (1986).

${ }^{14} \mathrm{P}$. West, Nucl. Phys. B 268, 113 (1986).

is X. Jaén, J. Llosa, and A. Molina, Phys. Rev. D 34, 2302 (1986).

${ }^{16} \mathrm{~F}$. Neyzi and Y. Nutku, J. Math. Phys. 28, 1499 (1987).

"M. Borneas, Anal. Univ. Timişoara 26, 37 (1988).

18 I. A. Batalin and G. A. Vilkovisky, Phys. Lett. B 102, 27 (1981).

${ }^{19} \mathrm{~K}$. Sundermeyer, Constrained Dynamics, Lecture Notes in Physics, Vol. 169 (Springer-Verlag, Berlin, 1982).

${ }^{20}$ R. Abraham and J. E. Marsden, Foundations of Mechanics (AddisonWesley, Reading, MA, 1978), 2nd ed.

${ }^{21}$ G. Marmo, N. Mukunda, and J. Samuel, Riv. Nuovo Cimento 6, 2 (1983).

22 M. J. Berguelt and E. A. De Kerf, Physica A 139, 101 (1986).

${ }^{23}$ J. F. Cariñena, C. López, and N. Román-Roy, J. Geom. Phys. 4, 315 (1987).
${ }^{24}$ X. Gràcia and J. M. Pons, Ann. Phys. (NY) 187, 355 (1988).

${ }^{25}$ M. V. Ostrogradskii, Mém. Ac. St.-Pétersbourg 6, 385 (1850).

${ }^{26}$ A. Pais and G. E. Uhlenbeck, Phys. Rev. 79, 145 (1950).

${ }^{27}$ M. Borneas, Phys. Rev. 186, 1299 (1969).

${ }^{28}$ D. M. Gitman, S. L. Ljahovič, and I. V. Tjutin, Izv. VUZ Fizika 8, 61 (1983).

${ }^{29}$ M. de León and P. R. Rodrigues, Generalized Classical Mechanics and Field Theory North-Holland Math. Studies, No. 112 (Elsevier, Amsterdam, 1985).

${ }^{30} \mathrm{~V}$. Tapia, Nuovo Cimento B 90, 15 (1985).

${ }^{31}$ V. V. Nesterenko, J. Phys. A: Math. Gen. 22, 1673 (1989).

${ }^{32} \mathrm{~J}$. Barcelos-Neto and N. R. F. Braga, "Higher order canonical formalism in field theory and constraints," Universidade Federal do Rio de Janeiro preprint IF/UFRJ/87/20.

${ }^{33}$ C. Batlle, J. Gomis, J. M. Pons, and N. Román-Roy, J. Phys. A: Math. Gen. 21, 2693 (1988).

${ }^{34}$ C. A. P. Galvão and N. A. Lemos, J. Math. Phys. 29, 1588 (1988).

${ }^{35}$ V. Tapia, Nuovo Cimento B 101, 183 (1988).

${ }^{36}$ J. M. Pons, Lett. Math. Phys. 17, 181 (1989).

${ }^{37}$ Y. Saito, R. Sugano, T. Ohta, and T. Kimura, J. Math. Phys. 30, 1122 (1989).

${ }^{38}$ W. M. Tulczyjew, C. R. Acad. Sci. Paris A 280, 1295 (1975).

${ }^{30}$ M. Crampin, W. Sarlet, and F. Cantrijn, Math. Proc. Cambridge Philos. Soc. 99, 565 (1986).

${ }^{40}$ M. de León and E. A. Lacomba, J. Phys. A: Math. Gen. 22, 3809 (1989).

${ }^{41}$ M. de León, A. Giraldo, and P. R. Rodrigues, "Sur la reduction de systèmes lagrangiens dégénérés d'ordre supérieur," preprint (1989).

${ }^{42}$ A. J. Hanson, T. Regge, and C. Teitelboim, Constrained Hamiltonian Systems (Accad. Naz. dei Lincei, Roma, 1976).

${ }^{43}$ C. Batlle, J. Gomis, J. M. Pons, and N. Román-Roy, J. Math. Phys. 27, 2953 (1986).

${ }^{44}$ C. Batlle, J. Gomis, J. M. Pons, and N. Román-Roy, Lett. Math. Phys. 13, 17 (1987).

${ }^{45}$ J. M. Pons, J. Phys. A: Math. Gen. 21, 2705 (1988).

${ }^{46}$ X. Gràcia and J. M. Pons, Lett. Math. Phys. 17, 175 (1989).

${ }^{47}$ R. Abraham, J. E. Marsden, and T. Ratiu, Manifolds, Tensor Analysis, and Applications (Addison-Wesley, Reading, MA 1983).

${ }^{48} \mathrm{~K}$. Yano and S. Ishihara, Tangent and Cotangent Bundles: Differential Geometry, Pure and Applied Mathematics, No. 16 (Dekker, New York, 1973).

${ }^{49}$ N. Bourbaki, Varietés Differentielles et Analytiques (Diffusion CCLS, Paris, 1983).

${ }^{50}$ J. Dieudonné, Elements d'Analyse (Gauthier-Villars, Paris, 1970), Vol. 3.

${ }^{51}$ D. Husemoller, Fibre Bundles Graduate Texts in Mathematics, Vol. 20 (Springer-Verlag, New York, 1975), 2nd ed.

${ }^{52}$ M. J. Gotay, J. M. Nester, and G. Hinds, J. Math. Phys. 19, 2388 (1978).

${ }^{53}$ D. J. Saunders and M. Crampin, J. Phys. A: Math. Gen. 23, 3169 (1990).

${ }^{54} \mathrm{~J}$. F. Cariñena and C. López, "The time evolution operator for higher order singular lagrangians," to appear in Int. J. Mod. Phys.

${ }^{55} \mathrm{X}$. Gràcia, "Sistemes dinàmics lligats: estudi geomètric i transformacions de gauge," Ph.D. thesis, Universitat de Barcelona, 1991.

${ }^{56}$ M. J. Gotay, and J. M. Nester, Ann. Inst. H. Poincaré A 32, 1 (1980).

${ }^{57}$ X. Gràcia, J. M. Pons, and N. Román-Roy, "Higher order conditions for singular lagrangian dynamics," Universitat de Barcelona preprint UBECM-PF 5/90

${ }^{58}$ P. A. M. Dirac, Lectures on Quantum Mechanics (Yeshiva Univ., New York, 1964).

${ }^{59}$ X. Gràcia and J. M. Pons, Int. J. Theor. Phys. 30, 511 (1991).

${ }^{60} \mathrm{X}$. Gràcia and J. M. Pons, "A generalized geometric framework for constrained systems," Universitat de Barcelona preprint UB-ECM-PF 7/90. 